\title{
On the Mosaic Method for Many-Dimensional Modal Logics: A Case Study Combining Tense and Modal Operators
}

\author{
Carlos Caleiro $^{1} \quad$ Luca Viganò $^{2} \quad$ Marco Volpe $^{1}$ \\ 1 SQIG, Instituto de Telecomunicações \\ Departamento de Matemática, Instituto Superior Técnico \\ Universidade Técnica de Lisboa, Portugal \\ \{ccal|mvolpe\}@math.ist.utl.pt \\ 2 Dipartimento di Informatica, Università di Verona, Italy \\ luca.vigano@univr.it
}

\begin{abstract}
We present an extension of the mosaic method aimed at capturing many-dimensional modal logics. As a proof-of-concept, we define the method for logics arising from the combination of linear tense operators with an "orthogonal" S5-like modality. We show that the existence of a model for a given set of formulas is equivalent to the existence of a suitable set of partial models, called mosaics, and apply the technique not only in obtaining a proof of decidability and a proof of completeness for the corresponding Hilbert-style axiomatization, but also in the development of a mosaic-based tableau system. We further consider extensions for dealing with the case when interactions between the two dimensions exist, thus covering a wide class of bundled Ockhamist branching-time logics, and present for them some partial results, such as a non-analytic version of the tableau system.
\end{abstract}

\section{Introduction}

The mosaic method has been introduced in algebraic logic as a way of proving decidability of the theories of some classes of algebras of relations [18,19]. The basic idea consists in showing that the existence of a model is equivalent to the existence of a (possibly finite) suitable set of fragments of models, called mosaics. The power of the method comes from the fact that, given a formula, one does not need to generate a full model in order to prove its satisfiability: it is enough to show that there exists such a set of mosaics. As a by-product, one obtains a decision procedure for the logic whenever such a (finite) set exists.

The mosaic method has been recently applied to prove decidability, complexity results and completeness of Hilbert-style axiomatizations for several modal logics $[14,17,29]$. With regard to temporal logics, a first work considering an adaptation of the technique to the linear temporal logic of general time is [16], including, as an application, the development of a mosaic-based semantic tableaux system, along with a method for automated theorem proving. The authors also 
discuss the generalization of these results to particular flows of time by suggesting possible modifications of the conditions defining mosaics and saturated sets of mosaics. Further works using mosaics in temporal logics established complexity results for the logic of until over general linear time [23] and the logic using both since and until over the reals [26] (see also [24,25] for more recent and general accounts on mosaics and complexity topics). In [21], a variant of the mosaic method has been used to prove decidability of a so-called temporal logic of parallelism, mentioned also in [27]. In [21], it is also shown that this logic does not enjoy (the usual form of) the finite model property and thus that the mosaic method is in some cases a more powerful tool for proving decidability.

In this paper, we consider the method described in [16] for linear-time logic as our starting point and propose an extension able to deal with logics arising from the combination of linear temporal operators with an "orthogonal" S5like modality. The resulting logic is described in [31] (in the case of general linear time). Alongside the linear-time mosaics (defined essentially as in [16]), which we will call vertical mosaics, we will consider also "orthogonal" horizontal mosaics. We will show that, as long as no interaction occurs between the two dimensions, the results of [16] extend to our case. Namely, we will prove that the existence of a model for a given set of formulas is equivalent to the existence of a suitable set of mosaics, and will apply the technique not only in obtaining a completeness proof for the corresponding Hilbert-style axiomatization, but also in the development of a mosaic-based tableau system. We will further show that a finite fragment of the language is enough for setting up the necessary sets of mosaics, thus obtaining a decision procedure for the logic, even a tableau-based one, as well as corresponding complexity bounds.

By following the classification described in [31], we will also define conditions modeling possible interactions between the two dimensions, thus covering a hierarchy of logics that culminate in the bundled Ockhamist branching temporal logic of general time. This logic corresponds to the logic of Kamp frames of [22], which differs from the logic of Ockhamist frames described in [31] only in the fact that the atomic harmony assumption, i.e., the evaluation of atomic formulas is given with respect to nodes of a tree and not to pairs (node, branch), is relaxed there. Though our mosaic definitions do not lead to a proof of decidability when interactions between the vertical and the horizontal components are considered, they still allow for giving non-analytic but interesting tableau systems for the logics. These tableau systems, inspired by the one described in [16] for linear-time, are strongly based on mosaics and are thus quite different from the standard semantic tableau systems for modal logics. The definition of the tableau rules follows very naturally from the mosaics definitions (each rule corresponding to a property that the mosaics are required to satisfy), and allows for an extremely clear and appealing representation of the (counter) model under construction. Indeed each node of a tableau can be seen as a snapshot of a fragment (at most six points) of such a model. Soundness and completeness are proved by exploiting the equivalence between the existence of a model and the existence of a saturated set of mosaics. We believe that these features make 
our tableau systems useful and appealing even in the cases for which analyticity could not be obtained, and for which some subsequent form of loop checking, to be better investigated, may perhaps be applied in order to retry decidability.

The treatment in the whole paper is strongly modular, both in terms of definitions and proofs, along the two dimensions, i.e., with respect to the possible restrictions applied to the linear order, e.g., density, discreteness, existence of starting/final points, and with respect to the interaction properties considered. Indeed, though many of the results presented here have been shown using other techniques, we believe that the mosaic method is interesting in itself as it provides a uniform way of establishing such metaproperties for large classes of logics.

We also remark that, although our focus here has been on a two-dimensional temporal logic, the approach is more generally suitable to the case of manydimensional modal logics [9] and seems to work well as long as possible extensions concern properties of a single dimension not interacting with the others. In the case of interactions between the components, only partial results are achieved and further work needs to be done. This seems to be related to analogous problems encountered in the field of combination of modal logics when considering transfer of results, e.g., finite axiomatizability or decidability, from the component logics to the combined one [15]. While such results are generally proved in the case of independent combinations of modal logics, e.g., in their fusion, very few general transfer results hold when their product is considered. Indeed the logics that we consider here are closely related to examples of products of epistemic and temporal/dynamic logics [5,13] and the commutativity-like property (weak diagram completion) that we will use in the next sections roughly corresponds to the perfect recall property of systems modeling the behavior of agents that "do not forget". Other examples of related logics are those based on the so-called $T \times W$ frames of Thomason [27,4,21]. These are more purely two-dimensional logics in the sense that the semantical structures are based on rectangular frames given by the product of a linear order $(T,<)$ with a given set $W$, on which an equivalence relation $\simeq_{t}$ is defined for each $t \in T$. The difference with respect to our frames is that there we have one single linear order and thus all the time-lines are "synchronized". Closer to the Ockhamist frames that we use are the Kamp frames presented in [27], where only the past of $\simeq$-related points is required to be synchronized.

We proceed as follows. In $\S 2$, we describe the logics of interest, and delimit the scope of applicability of our method. In $\S 3$, we define the mosaic method for the target class of logics and prove, modularly, that satisfiability is equivalent to the existence of a suitable set of vertical and horizontal mosaics. In $\S 4$, we consider applications of the method to the proof of completeness of Hilbert-style axiomatizations, to the formulation of mosaic-based tableau systems, and to the proof of decidability and complexity upper bounds. Finally, in $\S 5$, we discuss the pros and cons of the method, compare it with the literature, and propose some directions for future work. 


\section{Combinations of tense and modalities}

In this section, we present the logics that will be considered in the following; for further references, see [31,22]. The language consists of a set of classical connectives enriched by the linear temporal operators $\mathrm{G}$ and $\mathrm{H}$ and by the path quantifier $\forall$.

Definition 1. Given a denumerable set $\mathcal{P}$ of propositional symbols, with $p \in \mathcal{P}$, the set $\mathcal{F}$ of (well-formed) (Ockhamist) formulas is defined by the grammar

$$
A::=p|\neg A| A \wedge A|\mathrm{G} A| \mathrm{H} A \mid \forall A .
$$

The set of atomic formulas (or atoms) is $\mathcal{P}$. The complexity of a formula is the number of occurrences of connectives $(\neg, \wedge)$, operators $(\mathrm{G}, \mathrm{H})$ and path quantifiers $(\forall)$.

The intuitive meaning of $\mathrm{G}$ and $\mathrm{H}$ is always in the future and always in the past, respectively, with regard to a single branch. The path quantifier $\forall$ allows one to switch from a branch to another: intuitively, $\forall A$ holds at a node $s$ iff $A$ holds in all the branches starting from the node $s$. Derived connectives, operators and quantifiers (e.g., $\perp, \supset, \vee, F, P$ and $\exists$ ) are defined as is standard.

Our development and results are modular with respect both to the properties of the linear and the branching dimensions of the logics. Next, we settle their underlying linear temporal semantic structures.

Definition 2. $A$ (strict) linear order is a pair $(\mathcal{W}, \prec)$ where $\prec$ is a transitive and irreflexive relation on the non-empty set $\mathcal{W}$, such that for all $x, y \in \mathcal{W}$, if $x \neq y$ then either $x \prec y$ or $y \prec x$.

Other interesting properties of linear orders to be considered are:

(Fst) there exists $z \in \mathcal{W}$ such that for all $x \in \mathcal{W}, y \prec x$;

(Lst) there exists $y \in \mathcal{W}$ such that for all $x \in \mathcal{W}, x \prec y$;

(Nfst) for all $x \in \mathcal{W}$ there is $y \in \mathcal{W}$ such that $y \prec x$;

(Nlst) for all $x \in \mathcal{W}$ there is $y \in \mathcal{W}$ such that $x \prec y$;

(Dns) for all $x, y \in \mathcal{W}$, if $x \prec y$ then there is $z \in \mathcal{W}$ such that $x \prec z \prec y$;

(Udsc) for all $x, y \in \mathcal{W}$, if $y \prec x$ then there exists $z \in \mathcal{W}$ such that $z \prec x$ and there is no $u \in \mathcal{W}$ with $z \prec u \prec x$;

(Ddsc) for all $x, y \in \mathcal{W}$, if $x \prec y$ then there exists $z \in \mathcal{W}$ such that $x \prec z$ and there is no $u \in \mathcal{W}$ with $x \prec u \prec z$.

Fst/Lst guarantee the existence of a first/last (minimal/maximal) element, respectively. Reciprocally, Nfst/Nlst respectively guarantee that a first/last element does not exist. Dns guarantees that the order is dense. Finally, Udsc/Ddsc guarantee, respectively, downward/upward discreteness, that is, the existence of an immediate predecessor/successor for non-extremal elements.

Below, we will often confuse any meaningful (+ separated) sequence $\mathcal{C}$ of these properties with the class of all linear orders that satisfy the conditions in $\mathcal{C}$. Namely, we will use () to denote the class of all strict linear orders and 
(Dns+Fst+Nlst) to denote the class of all dense linear orders with a first element and without a final element.

Let us now introduce also the branching dimension.

Definition 3. A tree is an irreflexive ordered set $T=(\mathcal{W}, \prec)$ in which the set of the $\prec$-predecessors of any element of $\mathcal{W}$ is linearly ordered by $\prec$, that is, for all $x, y, z$ in $\mathcal{W}$, if $x \prec z$ and $y \prec z$ then either $x \prec y$ or $y \prec x$ or $x=y$.

$A$ path in a tree $T$ is a maximal linearly ordered set of nodes. A branch in a tree $T$ is any set of nodes $\{y \mid y \in \pi$ and $x \prec y\}$ for a given path $\pi$ and $a$ node $x \in \pi$. The least node $x$ of $a$ branch $b$ is the initial node of $b$. The set of all branches in $T$ will be denoted by $\mathcal{B} r(T)$. If $b$ and $c$ are branches and $b \subseteq c$, then we say that $b$ is a sub-branch of $c$ and $c$ is a super-branch of $b$.

Given a tree $T$, a bundle $B$ on $T$ is a subset of $\mathcal{B r}(T)$ closed under subbranches and super-branches and such that every node of $T$ belongs to some branch in $B$. A bundled tree is a pair $(T, B)$ where $T$ is a tree and $B$ is a bundle on $T$.

By following the terminology of [12], we can define the following classes of trees and bundled trees.

Definition 4. Let $\mathcal{C}$ be a class of linear orders. We define $\mathcal{T}(\mathcal{C})$ as the class of all trees in which every path is in $\mathcal{C}, \mathcal{B}(\mathcal{C})$ as the class of bundled trees $(T, B)$ such that $T \in \mathcal{T}(\mathcal{C}), \mathcal{B}^{+}(\mathcal{C})$ as the class of all bundled trees $(T, B)$ such that every path in the bundle $B$ is in $\mathcal{C}$.

The semantics of branching-time logics is commonly defined on the tree-like structures given above (we refer the reader to, e.g., [31] for a rigorous presentation). However, when considering bundled trees, such a semantics can be given in a more traditional Kripkean style by considering the so-called Ockhamist frames [31] (closely related to the Kamp frames of [27]), i.e., triples of the form $(\mathcal{W}, \prec, \simeq)$, in which $\mathcal{W}$ corresponds to the set of branches of the (bundled) tree, $\prec$ is the inclusion relation between branches and $\simeq$ is the equivalence relation of having the same initial node, as illustrated by Fig. 1.

Definition 5. Let $\mathcal{C}$ be a class of linear orders. $A \mathcal{C}$-basic-frame is a triple $(\mathcal{W}, \prec$ $, \simeq)$, where $(\mathcal{W}, \prec)$ is a non-empty union of linear orders in $\mathcal{C}$ and $\simeq$ is an equivalence relation on $\mathcal{W}$.

Other interesting properties of frames to be considered are:

(Dsj) for all $x, y \in \mathcal{W}$, if $x \simeq y$ then $x \nprec y$;

(Wdc) for all $x, y, y^{\prime} \in \mathcal{W}$, if $x \prec y \simeq y^{\prime}$ then there exists $x^{\prime} \in \mathcal{W}$ such that $x \simeq x^{\prime} \prec y^{\prime}$;

(Sdc) for all $x, y, z, x^{\prime}, z^{\prime} \in \mathcal{W}$, if $x \prec y \prec z \simeq z^{\prime}$ and $x \simeq x^{\prime} \prec z^{\prime}$ then there exists $y^{\prime} \in \mathcal{W}$ such that $y^{\prime} \simeq y$ and $x^{\prime} \prec y^{\prime} \prec z^{\prime}$;

$(\mathrm{Mb})$ for all $x, y \in \mathcal{W}$, if $x \simeq y$ and $x \neq y$, then there exists $x^{\prime} \in \mathcal{W}$ such that $x^{\prime} \succ x$ and there is no $y^{\prime} \in \mathcal{W}$ with $y^{\prime} \succ y$ and $x^{\prime} \simeq y^{\prime}$;

$\left(\mathrm{Mb}^{-}\right)$for all $x, y \in \mathcal{W}$, if $x$ is $\prec$-maximal and $x \simeq y$ then $x=y$. 

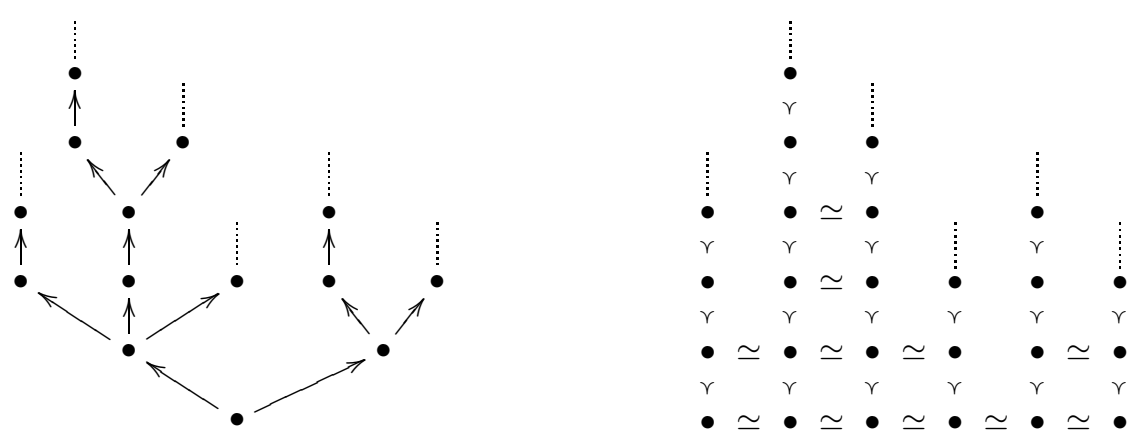

Fig. 1. A bundled tree (left) and the corresponding Ockhamist frame (right).

The property Dsj stands for disjointness of $\prec$ and $\simeq$, and comes from the fact that a node in a tree cannot be a descendant of itself. Wdc stands for weak diagram completion and is a consequence of the linearity of the order relation in a tree. Sdc is a strong form of Wdc and stands for strong diagram completion. Finally, the maximality of branches condition $\mathrm{Mb}$ models the fact that two distinct branches in a tree must have disjoint sub-branches. $\mathrm{Mb}^{-}$is another way of expressing the maximality of branches.

Below, we will often confuse any meaningful (+ separated) sequence $\mathcal{D}$ of these properties with the class of all basic frames that satisfy the conditions in $\mathcal{D}$. Given a class $\mathcal{C}$ of linear orders, the elements of such a class will be dubbed $\mathcal{C}$-D-frames. The class of Ockhamist frames is usually defined to be the class of ()-(Dsj+Wdc+Mb)-frames.

As is standard, we obtain an interpretation structure for the logical language by providing a frame with a valuation function.

Definition 6. A $\mathcal{C}$-D-structure is a 4-tuple $(\mathcal{W}, \prec, \simeq, \mathcal{V})$, where $(\mathcal{W}, \prec, \simeq)$ is a $\mathcal{C}$-D-frame and $\mathcal{V}$ is a valuation function $\mathcal{V}: \mathcal{W} \rightarrow 2^{\mathcal{P}}$, where $\mathcal{P}$ is the set of propositional symbols.

In the literature, the semantics of Ockhamist branching-time logics is sometimes defined by requiring that the valuation function obeys particular conditions, e.g., as in [22], that points $\simeq$-related satisfy the same set of atoms (we will sometimes refer to this assumption as atomic harmony). We will see below how our treatment can be adapted in order to deal with this case too.

The notion of truth with respect to a point in a structure is now easily definable, having the temporal operators $\mathrm{G}$ and $\mathrm{H}$ operate along the $\prec$-lines of points, and the quantifier $\forall$ within $\simeq$-equivalence classes.

Definition 7. The satisfaction relation $\models$ for Ockhamist formulas over a $\mathcal{C}-\mathcal{D}$ structure $M=(\mathcal{W}, \prec, \simeq, \mathcal{V})$ and a point $u \in \mathcal{W}$ is defined by: 


$$
\begin{array}{lll}
M, u \models p & \text { iff } & p \in \mathcal{V}(u) ; \\
M, u=\neg A & \text { iff } & M, u \models A ; \\
M, u=A \wedge B & \text { iff } & M, u \models A \text { and } M, u \models B ; \\
M, u \models \mathrm{G} A & \text { iff } & M, v \models A \text { for all } v \text { such that } u \prec v ; \\
M, u=\mathrm{H} A & \text { iff } & M, v \models A \text { for all } v \text { such that } v \prec u ; \\
M, u \models \forall A & \text { iff } & M, v \models A \text { for all } v \text { such that } u \simeq v .
\end{array}
$$

This notion of $\mathcal{C}-\mathcal{D}$ satisfaction extends to the notions of $\mathcal{C}$-D $D$-satisfiability, $\mathcal{C}$ $\mathcal{D}$-validity and $\mathcal{C}$ - $\mathcal{D}$-entailment as is standard.

Below, we will write $\mathcal{L}(\mathcal{C}, \mathcal{D})$ to refer to the logic on the Ockhamist language defined by the class of all $\mathcal{C}$ - $\mathcal{D}$-structures.

Lemma 1. Let $\mathcal{C}$ be a class of linear orders. Then:

(i) $\mathcal{L}(\mathcal{C},())=\mathcal{L}(\mathcal{C},(D s j))$;

(ii) $\mathcal{L}(\mathcal{C},(D s j+W d c))=\mathcal{L}(\mathcal{C},(W d c+S d c))$;

(iii) $\mathcal{L}(\mathcal{C},(D s j+W d c+M b))=\mathcal{L}\left(\mathcal{C},\left(W d c+S d c+M b^{-}\right)\right)$and both coincide also with the logic defined over bundled trees in the class $\mathcal{B}^{+}(\mathcal{C})$.

Proof. (i), (ii) and the first equivalence in (iii) can be shown by a trivial adaptation of analogous results proved in [31] in the case of general linear-time. We obtain the last equivalence by noticing that there is a one-to-one correspondence between elements of $\mathcal{B}^{+}(\mathcal{C})$, for a given class $\mathcal{C}$ of linear orders, and Ockhamist frames in which every linear component is in $\mathcal{C}$.

Given these equivalences, from now on we will focus on the $\operatorname{logics} \mathcal{L}(\mathcal{C}, \mathcal{D})$ where $\mathcal{D}$ is one of our four target branching classes, that is: (), (Wdc), (Wdc+Sdc) or $\left(\mathrm{Wdc}+\mathrm{Sdc}+\mathrm{Mb}^{-}\right)$. This will allow us to span from the logic of basic frames toward the logic of Ockhamist frames in a stepwise manner.

Moreover, though in the rest of the paper our reference, for what concerns the semantical structures, will be the $\mathcal{C}$ - $\mathcal{D}$-frames of Definition 6 , Lemma 1 will allow us to read our results also in terms of tree-like structures. In particular, by Lemma 1, the final point of our hierarchy of branching classes (the one given by the combination $\left(W d c+S d c+M b^{-}\right)$) corresponds, for $\mathcal{C}$ some class of linear orders, to the class $\mathcal{B}^{+}(\mathcal{C})$ of Definition 4 . Notice, however, that many of the classes of linear orders $\mathcal{C}$ considered here, i.e., $\mathcal{C}=(), \mathcal{C}=(F s t), \mathcal{C}=(D d s c)$, $\mathcal{C}=(U d s c)$ and $\mathcal{C}=(D n s)$, enjoy closure properties such that the classes $\mathcal{B}(\mathcal{C})$ and $\mathcal{B}^{+}(\mathcal{C})$ coincide ${ }^{3}$; see [12] for further details and a proof of this fact. Thus, for such particular $\mathcal{C}$ s, our results indeed extend also to the logic defined by the class $\mathcal{B}(\mathcal{C})$.

\footnotetext{
${ }^{3}$ In particular, $\mathcal{B}(())=\mathcal{B}^{+}(())$implies that the logic $\mathcal{L}\left((),\left(W d c+S d c+M b^{-}\right)\right)$coincides with the Ockhamist logic of general time over bundled trees described in [31].
} 


\section{The mosaic method}

In this section, we give an extension of the definition of the mosaic method for a linear tense logic, given in [16], to the case when an orthogonal modality is introduced. By considering some interaction properties, a class of bundled branching-time logics is covered.

Intuitively, the linear temporal (vertical, in our terminology) mosaics of [16] can be seen as pairs $(\Gamma, \Delta)$ where $\Gamma$ and $\Delta$ refer to two points in a temporal structure, such that the point associated to $\Gamma$ precedes (by the relation $\prec$ ) the one associated to $\Delta$. $\Gamma$ and $\Delta$ are indeed sets of formulas, namely formulas that are satisfied at the corresponding point. Given this basic intuition, it is reasonable to require that linear temporal mosaics satisfy some local coherence conditions: as an example, given a mosaic $(\Gamma, \Delta)$, we want that if $\mathrm{G} A \in \Gamma$, then $A \in \Delta$. Moreover, we are interested in considering particular sets of mosaics, saturated in such a way that we are able to build a complete model by just composing the mosaics contained in a given set of that kind. In other words, we need to define the saturation conditions that a "good" set of mosaics is required to satisfy. Basically, this amounts to making sure that each counterexample occurring in the model we are building can be "cured". In the context of linear tense logics, a counterexample consists in the presence of a point $w$ labeled with a formula of the form $\mathrm{F} A$ such that all of its successors are labeled with $\neg A$. By "curing" it, we mean adding a new point $w^{\prime}$ to the structure (as a successor of $w)$ such that the labeling set of $w^{\prime}$ contains $A$.

We keep here the intuition behind linear temporal mosaics [16] but need to consider also horizontal mosaics, to take into account the branching nature of the logics. Expectedly, these will be pairs $(\Gamma, \Delta)$ of sets of formulas, where the sets now refer to $\simeq$-related points in the structure. Corresponding coherence and saturation conditions will apply. Namely such a compatibility will consist primarily in requiring as a coherence condition that $\Gamma$ and $\Delta$ agree with respect to state formulas (which must include the propositional symbols in case we adopt the atomic harmony approach). Saturation-wise, there is also the need to deal with "branching counterexamples", i.e., points labeled with a formula of the form $\exists A$ such that no $\simeq$-related point contains $A$. Of course, further requirements need to be satisfied in order to cover all the necessary properties, namely regarding the interaction between horizontal and vertical mosaics.

\subsection{Mosaics}

In the most general case, that is, when, as in Definition 6, no particular assumptions are made on the way atoms are evaluated, it is straightforward to check that the set of state formulas can be defined recursively as follows:

1. if $A$ is a formula, then $\forall A$ is a state formula;

2. if $A$ and $B$ are state formulas, then $A \wedge B$ is a state formula;

3 . if $A$ is a state formula, then $\neg A$ is a state formula. 
If we are interested in logics where the evaluation of atoms depends only on the state (and not on the particular path), i.e., if $v \simeq w$ implies $\mathcal{V}(v)=\mathcal{V}(w)$, then the following further base case needs to be added to the conditions above:

0 . if $A$ is an atomic proposition, then $A$ is a state formula.

In any case, it is clear that satisfaction at any $\simeq$-related points in an interpretation structure agrees on state formulas.

The following definitions are essential in supporting the construction of sets of mosaics based not necessarily on the whole Ockhamist language $\mathcal{F}$, but on suitable (possibly finite) sublanguages $\Lambda \subseteq \mathcal{F}$. Below, unless otherwise stated we consider fixed such a set $\Lambda$. As a minimal requirement, we will assume that $\Lambda$ is closed under subformulas and single negation (of non-negated formulas).

Definition 8. Let $\Gamma, \Delta \subseteq \Lambda$. We say that $\Gamma$ and $\Delta$ are $\Lambda$-state-equivalent, and we write $\Gamma \sim_{\Lambda} \Delta$, if for each state formula $A \in \Lambda, A \in \Gamma$ if and only if $A \in \Delta$.

Definition 9. A point (on $\Lambda$ ) is a set of formulas $\Gamma \subseteq \Lambda$ satisfying the following local conditions:

for every formula $A \in \Lambda$,

(L1) $A \in \Gamma$ iff $\neg A \notin \Gamma$;

(L2) $A=B \wedge C \in \Gamma$ iff $\{B, C\} \subseteq \Gamma$;

(L3) if $A=\forall B \in \Gamma$ then $B \in \Gamma$.

$A$ point $\Gamma$ is further said to be $e^{4}$ :

- future unbounded (or a FU-point) if $\mathrm{F} \top \in \Gamma$;

- future bounded (or a $\mathrm{FB}$-point) if $(\mathrm{FG} \perp) \vee(\mathrm{G} \perp) \in \Gamma$;

- past unbounded (or a $\mathrm{PU}$-point) if $\mathrm{P} \top \in \Gamma$;

- past bounded (or a $\mathrm{PB}$-point) if $(\mathrm{PH} \perp) \vee(\mathrm{H} \perp) \in \Gamma$.

Definition 10. A mosaic (on $\Lambda$ ) is a pair $(\Gamma, \Delta)$ or just $(\Gamma)$, where $\Gamma$ and $\Delta$ are points on $\Lambda$. We say that a mosaic $(\Gamma, \Delta)$ is a vertical mosaic iff it satisfies the following vertical coherence conditions:

for every formula $A \in \Lambda$,

(V1) if $A=\mathrm{G} B \in \Gamma$ then $B \in \Delta$;

(V2) if $A=\mathrm{H} B \in \Delta$ then $B \in \Gamma$;

(V3) if $A=\mathrm{G} B \in \Gamma$ then $\mathrm{G} B \in \Delta$;

(V4) if $A=\mathrm{H} B \in \Delta$ then $\mathrm{H} B \in \Gamma$.

We say that a mosaic $(\Gamma, \Delta)$ is a horizontal mosaic iff it satisfies the following horizontal coherence condition:

(H1) $\Gamma$ and $\Delta$ are $\Lambda$-state-equivalent.

\footnotetext{
${ }^{4}$ Notice that the definitions of future/past (un)boundedness require, mutatis mutandis, that the corresponding formulas $\mathrm{F} T,(\mathrm{FG} \perp) \vee(\mathrm{G} \perp), \mathrm{P} \top,(\mathrm{PH} \perp) \vee(\mathrm{H} \perp) \in \Lambda$.
} 
A singular mosaic $(\Gamma)$ is both a vertical and a horizontal mosaic.

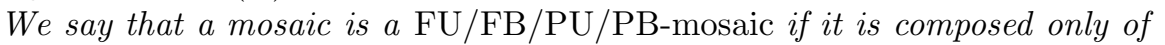
$F U / F B / P U / P B$-points, respectively.

Let us now consider sets of mosaics.

Definition 11. The set of points of a set of mosaics $S$ (on $\Lambda$ ) is the set Points $(S)=$ $\{\Omega \subseteq \Lambda \mid(\Omega) \in S$ or there exists $(\Gamma, \Delta) \in S$ with $\Omega=\Gamma$ or $\Omega=\Delta\}$.

Vertical mosaics are subject to the following saturation properties.

Definition 12. A set $S$ of vertical mosaics (on $\Lambda$ ) is a ()-vertically saturated set of mosaics (on $\Lambda$ ) (a ()-VSSM for short) if it satisfies the following ()-vertical saturation conditions:

for every $\Omega \in \operatorname{Points}(S)$,

(SV1) if $\mathrm{F} A \in \Omega$ then there exists $(\Omega, \Gamma) \in S$ with $A \in \Gamma$;

(SVD) if $\mathrm{P} A \in \Omega$ then there exists $(\Gamma, \Omega) \in S$ with $A \in \Gamma$;

for every mosaic $(\Gamma, \Delta) \in S$,

(SV3) if $\mathrm{F} A \in \Gamma$, then:

(i) $A \in \Delta$ or $\mathrm{F} A \in \Delta$; or

(ii) there exist $(\Gamma, \Omega),(\Omega, \Delta) \in S$ with $A \in \Omega$;

$\left(S V_{4}\right)$ if $\mathrm{P} A \in \Delta$, then:

(i) $A \in \Gamma$ or $\mathrm{P} A \in \Gamma$; or

(ii) there exist $(\Gamma, \Omega),(\Omega, \Delta) \in S$ with $A \in \Omega$.

Additional vertical saturation conditions of interest are:

for every mosaic $(\Gamma, \Delta) \in S$,

(SVDns) there exists $\Omega \in \operatorname{Points}(S)$ such that $(\Gamma, \Omega),(\Omega, \Delta) \in S$;

(SVUdsc) if $\mathrm{F} A \in \Gamma$, then:

(i) $A \in \Delta$ or $\mathrm{F} A \in \Delta$; or

(ii) there exist $(\Gamma, \Omega),(\Omega, \Delta) \in S$ with $\{A, \neg \mathrm{F} A\} \subseteq \Omega$;

(SVDdsc) if $\mathrm{P} A \in \Delta$, then:

(i) $A \in \Gamma$ or $\mathrm{P} A \in \Gamma$; or

(ii) there exist $(\Gamma, \Omega),(\Omega, \Delta) \in S$ with $\{A, \neg \mathrm{P} A\} \subseteq \Omega$.

Given a class $\mathcal{C}$ of linear structures, $S$ is said to be $\mathcal{C}$-vertically saturated (a $\mathcal{C}$-VSSM for short) if $S$ is a ()-VSSM that further satisfies the following conditions, corresponding to each property in $\mathcal{C}$ :

- Fst/Lst/Nfst/Nlst correspond to requiring that $S$ is a set of $P B / F B / P U / F U$ mosaics, respectively;

- Dns corresponds to requiring that SVDns holds;

- Udsc/Ddsc correspond to requiring SVUdsc/SVDdsc hold, respectively.

Horizontal mosaics are also subject to saturation properties. 
Definition 13. A set $S$ of horizontal mosaics (on $\Lambda$ ) is a horizontally saturated set of mosaics (on $\Lambda$ ) (a HSSM for short) if it satisfies the following horizontal saturation condition:

for every $\Omega \in \operatorname{Points}(S)$,

(SH1) if $\exists A \in \Omega$ and $A \notin \Omega$ then there exists $\Gamma \in \operatorname{Points}(S)$ such that $(\Omega, \Gamma) \in$ $S$ and $A \in \Gamma$.

We now need to consider the joint effect of vertical and horizontal mosaics.

Definition 14. Let $\mathcal{C}$ be a class of linear orders. $A \mathcal{C}$-basic-structure of mosaics is a pair $S=\left(S_{V}, S_{H}\right)$ such that $S_{V}$ is a $\mathcal{C}-V S S M, S_{H}$ is an HSSM, and Points $\left(S_{V}\right)=\operatorname{Points}\left(S_{H}\right)$. The set of points of the structure of mosaics $\mathrm{S}$ is precisely Points $(S)=\operatorname{Points}\left(S_{V}\right)=\operatorname{Points}\left(S_{H}\right)$.

Additional combined conditions of interest are:

(SWdc) if $(\Omega, \Gamma) \in S_{V}$ and $(\Gamma, \Delta) \in S_{H}$ then there exists $\Phi \in \operatorname{Points}(S)$ such that $(\Phi, \Delta) \in S_{V}$ and $(\Omega, \Phi) \in S_{H}$;

(STrn) if $(\Gamma, \Delta),(\Delta, \Omega) \in S_{V}$ then $(\Gamma, \Omega) \in S_{V}$;

(SCon) if $(\Gamma, \Omega),(\Delta, \Omega) \in S_{V}$ then either $\Gamma=\Delta$, or $(\Gamma, \Delta) \in S_{V}$ or $(\Delta, \Gamma) \in$ $S_{V} ;$

$(S S d c)$ if $(\Phi, \Omega),(\Omega, \Gamma),(\Psi, \Delta) \in S_{V}$ and $(\Phi, \Psi),(\Gamma, \Delta) \in S_{H}$ then there exists $\Upsilon \in$ Points $(S)$ such that $(\Psi, \Upsilon),(\Upsilon, \Delta) \in S_{V}$ and $(\Omega, \Upsilon) \in S_{H}$;

$\left(S M b^{-}\right)$if $(\Gamma, \Delta) \in S_{H}$ and $\mathrm{G} \perp \in \Gamma$ then $\Gamma=\Delta^{5}$.

Given one of the target branching classes $\mathcal{D}, S=\left(S_{V}, S_{H}\right)$ is said to be a $\mathcal{C}$ $\mathcal{D}$-structure of mosaics if $S$ is a $\mathcal{C}$-basic-structure of mosaics that further satisfies the following conditions, corresponding to each class $\mathcal{D} \neq()$ :

$-\mathcal{D}=(W d c)$ requires that $S W d c, S T r n$ and SCon hold;

$-\mathcal{D}=(W d c+S d c)$ requires that $S W d c$ and $S S d c$;

- $\mathcal{D}=\left(W d c+S d c+M b^{-}\right)$requires $S W d c, S S d c$ and $S M b^{-}$to hold.

Given a structure of mosaics $S$ and a set of formulas $\Gamma$, we say that $S$ is a structure of mosaics for $\Gamma$ if there exists $\Omega \in \operatorname{Points}(S)$ such that $\Gamma \subseteq \Omega$.

\subsection{Mosaics and satisfiability}

We will now show that the existence of a saturated set of mosaics for a given set of formulas corresponds to the existence of a model for such a set.

Definition 15. Let $F=(\mathcal{W}, \prec, \simeq)$ be a frame. $A$ chronicle for $F$ on $\Lambda$ is a function $\delta$ assigning a subset of $\Lambda$ to every element of $\mathcal{W}$ such that the following conditions are satisfied: for every $v, v^{\prime} \in \mathcal{W}$,

(i) $A \in \delta(v)$ iff $\neg A \notin \delta(v)$;

(ii) $A \wedge B \in \delta(v)$ iff $\{A, B\} \subseteq \delta(v)$;

\footnotetext{
${ }^{5}$ Notice that the $\mathrm{SMb}^{-}$condition only makes sense if we require that $\mathrm{G} \perp \in \Lambda$.
} 
(iii) if $\forall A \in \delta(v)$, then $A \in \delta(v)$;

(iv) if $\mathrm{G} A \in \delta(v)$ and $v \prec v^{\prime}$, then $\{A, \mathrm{G} A\} \subseteq \delta\left(v^{\prime}\right)$;

(v) if $\mathrm{H} A \in \delta(v)$ and $v^{\prime} \prec v$, then $\{A, \mathrm{H} A\} \subseteq \delta\left(v^{\prime}\right)$;

(vi) if $v \simeq v^{\prime}$ then $\delta(v) \sim_{\Lambda} \delta\left(v^{\prime}\right)$.

We say that a chronicle $\delta$ is based on a structure of mosaics $S=\left(S_{V}, S_{H}\right)$, defined on the same $\Lambda$, if:

(vii) if $v \prec v^{\prime}$ and there is no $v^{\prime \prime}$ such that $v \prec v^{\prime \prime} \prec v^{\prime}$, then $\left(\delta(v), \delta\left(v^{\prime}\right)\right) \in$ $S_{V}$;

(viiii) if $v \simeq v^{\prime}$, then there exist $v_{1} \simeq \ldots \simeq v_{n}$ such that $v=v_{1}, v^{\prime}=v_{n}$ and $\left(\delta\left(v_{i}\right), \delta\left(v_{i+1}\right)\right) \in S_{H}$ for $0<i<n$.

Let $\mathcal{D}$ be a target branching class and $S$ be a structure of mosaics. Given a $\mathcal{C}$-D-frame $F$ and a chronicle $\delta$ for it, the pair $(F, \delta)$ will be referred to as a $\mathcal{C}$-D-chronicled frame based on $S$.

Conditions $(i)-(v i)$ are the analogous of the coherence conditions in the definition of vertical and horizontal mosaics. Conditions (vii) and (viii) ensure that the chronicle is built out of mosaics from a given structure.

Definition 16. Let $F=(\mathcal{W}, \prec, \simeq)$ be a ()-()-frame, $v \in \mathcal{W}, \delta$ a chronicle for $F$ and $A$ a formula. An element $\langle v, \mathrm{~F} A\rangle$ is a vertical future defect of $(F, \delta)$ if:

(i) $\mathrm{F} A \in \delta(v)$; and

(ii) (ii) for every $v^{\prime} \in \mathcal{W}$ such that $v \prec v^{\prime}$, we have $A \notin \delta\left(v^{\prime}\right)$.

$\langle v, \mathrm{P} A\rangle$ is a vertical past defect of $(F, \delta)$ if:

(i) $\mathrm{P} A \in \delta(v)$; and

(ii) for every $v^{\prime} \in \mathcal{W}$ such that $v^{\prime} \prec v$, we have $A \notin \delta\left(v^{\prime}\right)$.

Finally, $\langle v, \exists A\rangle$ is a horizontal defect of $(F, \delta)$ if:

(i) $\exists A \in \delta(v)$; and

(ii) for every point $v^{\prime} \in \mathcal{W}$, if $v \simeq v^{\prime}$ then $A \notin v^{\prime}$.

Lemma 2. Let $\mathcal{D}$ be a target branching class in $\{(),(W d c),(W d c+S d c)\}, S=$ $\left(S_{V}, S_{H}\right)$ a ()-D-structure of mosaics, $(F=(\mathcal{W}, \prec, \simeq), \delta)$ a finite () -D -chronicled frame based on $S$ and $\alpha$ a defect on $(F, \delta)$. Then there exists a finite ()-D chronicled frame based on $S$ that extends $(F, \delta)$ and such that $\alpha$ is not a defect in it.

Proof. The proof proceeds by showing how to cure vertical and horizontal defects and how to guarantee that the resulting frame is still of the required type. Notice that if $\mathcal{D} \neq()$, the procedure may require the addition of more than one point.

Curing of vertical defects. Vertical defects are cured in the same way as described in [16]. We recall the case of a linear future defect; the treatment of past defects is just symmetrical. Let $\alpha$ be $\langle v, \mathrm{~F} A\rangle$ for some $v \in \mathcal{W}$ and some formula $A$. We can consider a $v^{\prime}$ that is the $\prec$-maximal element of $\mathcal{W}$ such that $\mathrm{F} A \in v^{\prime}$. Since $d$ is a defect of $(F, \delta)$, such a $v^{\prime}$ exists. By curing the defect $\left\langle v^{\prime}, \mathrm{F} A\right\rangle$, we will also cure all the defects $\langle w, \mathrm{~F} A\rangle$ for $w \prec v^{\prime}$. We have two subcases: 
(a) $v^{\prime}$ is the greatest element of $\mathcal{W}$ according to $\prec$. Then, by the saturation condition SV1, there is a mosaic $\left(\Gamma_{0}^{\prime}, \Gamma_{1}^{\prime}\right)$ in $S$ such that $\Gamma_{0}^{\prime}=\delta\left(v^{\prime}\right)$ and $A \in \Gamma_{1}^{\prime}$. We can define a new frame $F^{\prime}=\left\{\mathcal{W}^{\prime}, \prec^{\prime} \simeq^{\prime}\right\}$ obtained by adding a new element $v^{\prime \prime}$ labeled with $\Gamma_{1}^{\prime}$. Namely, we define $\mathcal{W}^{\prime}=\mathcal{W} \cup\left\{v^{\prime \prime}\right\}, \prec^{\prime}$ as the smallest linear order relation containing $\prec$ and such that $w \prec v^{\prime \prime}$ for every $w \in \mathcal{W}$ and $\simeq^{\prime}=\simeq \cup\left\{\left(v^{\prime \prime}, v^{\prime \prime}\right)\right\}$. We can associate to $F^{\prime}$ a chronicle $\delta^{\prime}$ that extends $\delta$ by assigning $\Gamma_{1}^{\prime}$ to $v^{\prime \prime}$.

(b) $v^{\prime}$ is not the $\prec$-greatest element of $\mathcal{W}$. Then, there exists an element $v^{\prime \prime} \in \mathcal{W}$ such that $v^{\prime \prime}$ is the immediate successor of $v^{\prime}$, according to the relation $\prec$, and, by the maximality of $v^{\prime}, \neg \mathrm{F} A \in \delta\left(v^{\prime \prime}\right)$. By the condition SV3, there exist two mosaics $\left(\Delta_{0}, \Delta\right),\left(\Delta, \Delta_{1}\right) \in S$ such that $\Delta_{0}=\delta\left(v^{\prime}\right)$, $\Delta_{1}=\delta\left(v^{\prime \prime}\right)$ and $A \in \Delta$. Then we define an extension $F^{\prime}=\left\{\mathcal{W}^{\prime}, \prec^{\prime}, \simeq^{\prime}\right\}$, by inserting a point $v^{*}$ between $v^{\prime}$ and $v^{\prime \prime}$. A chronicle $\delta^{\prime}$ for $F^{\prime}$ is obtained by extending $\delta$ with the assignment $\delta^{\prime}\left(v^{*}\right)=\Delta$.

Curing of horizontal defects. If $\alpha$ is a horizontal defect, i.e., $A=\exists A^{\prime}$ for some $A^{\prime}$, then, by the saturation condition SH1, we know that there exists $\left(\Delta, \Delta^{\prime}\right) \in$ $S_{H}$ such that $\delta(v)=\Delta$ and $A \in \Delta^{\prime}$. Then we define a frame $F^{\prime}=\left(\mathcal{W}^{\prime}, \prec^{\prime}\right.$ ,$\left.\simeq^{\prime}\right)$, where $\mathcal{W}^{\prime}=\mathcal{W} \cup\left\{v^{\prime}\right\}, \prec^{\prime}=\prec$ and $\simeq^{\prime}$ is the reflexive, symmetric and transitive closure of $\simeq \cup\left\{\left(v, v^{\prime}\right)\right\}$. We can associate to $F^{\prime}$ a chronicle $\delta^{\prime}$ that extends $\delta$ by assigning $M^{\prime}$ to $v^{\prime}$.

Preserving relational properties. According to the procedure described above, curing a defect on a ()-(Wdc)-chronicled frame may produce a chronicled frame that is not of the same type. Namely, the curing of $\alpha$ could generate in the new frame $F^{\prime}=\left(\mathcal{W}^{\prime}, \prec^{\prime}, \simeq^{\prime}\right)$ a counterexample to the property $\mathrm{Wdc}$, i.e., three points $v_{1}, w_{1}$ and $w_{1}^{\prime}$ in $\mathcal{W}^{\prime}$ such that $v_{1} \prec^{\prime} w_{1} \simeq^{\prime} w_{1}^{\prime}$ but such that there is no point $v_{1}^{\prime}$ in $\mathcal{W}^{\prime}$ for which $v_{1} \simeq^{\prime} v_{1}^{\prime}$ and $v_{1}^{\prime} \prec^{\prime} w_{1}^{\prime}$ hold. Such situations, which will be referred to as Wdc-defects, need to be repaired in a different way according to the fact that $\mathcal{D}$ also contains Sdc or not.

(a) Let $\mathcal{D}=(W d c)$. Since $\left(F^{\prime}, \delta^{\prime}\right)$ is based on $S$, by the transitive closure ensured by property STrn and by condition (vii) in Definition 15, there exists $\left(\delta^{\prime}\left(v_{1}\right), \delta^{\prime}\left(w_{1}\right)\right) \in S_{V}$. Moreover, by condition (viii) in Definition 15 , there exists a sequence $w_{1} \simeq \ldots \simeq w_{n}$ such that $w_{n}=w_{1}^{\prime}$ and $\left(\delta^{\prime}\left(w_{i}\right), \delta^{\prime}\left(w_{i+1}\right)\right) \in S_{H}$ for $0<i<n$. By construction, for $0<i<n$, the sequence $v_{1} \prec^{\prime} w_{1} \simeq^{\prime} w_{i}$ also represents a Wdc-defect. All such defects will be cured by applying, in turn, to all the $0<i<n$, the following procedure. Let $v_{i}$ be the last point added to cure a defect (as a base case it will coincide with $\left.v_{1}\right)$ and $\left(F^{\prime \prime}, \delta^{\prime \prime}\right)$ the chronicled frame obtained as a result of the $(i-1)$-th step $\left(\delta^{\prime \prime}=\delta^{\prime}\right.$, if $\left.i=1\right)$. Then the sequence $v_{i} \prec$ ' $w_{i} \simeq^{\prime \prime} w_{i+1}$ is a Wdc-defect. As a result of the $(i-1)$-th step (or by hypothesis, if $i=1$ ) we have $\left(\delta^{\prime \prime}\left(v_{i}\right), \delta^{\prime \prime}\left(w_{i}\right)\right) \in S_{V}$. Then, by the saturation condition SWdc on $S$, we know that there exists a point $\Delta \in \operatorname{Points}(S)$ such that $\left(\Delta, \delta^{\prime \prime}\left(w_{i+1}\right)\right) \in S_{V}$ and $\left(\delta^{\prime \prime}\left(v_{i}\right), \Delta\right) \in S_{H}$. It is then possible to build a new frame by extending $F^{\prime \prime}$ with a further point $v_{i+1}$ and associate to it a proper chronicle that is the extension of $\delta^{\prime \prime}$ assigning $\Delta$ to $v_{i+1}$. Condition SCon allows for positioning the new point properly along the $\prec$ "-order. Such a procedure will eventually cure 
all the defects in the sequence and thus also the one related to the triple $\left(v_{1}, w_{1}, w_{1}^{\prime}\right)$.

(b) Now let $\mathcal{D}=(W d c+S d c)$. There are three possibilities: $(i)$ the Wdcdefects arise from the curing of a vertical defect made by inserting a point in the middle of a linear order (case $(b)$ in the curing of a vertical defect above); (ii) the Wdc-defects arise from the curing of a vertical past defect occurring at a point at the bottom of a linear order; or (iii) the Wdc-defects arise from the curing of a horizontal defect. In the case $(i)$, we notice that the possible Wdc-defects are also counterexamples to Sdc, since Wdc and Sdc combined give the frame the shape of a (partial) grid, with $\prec$ operate vertically and $\simeq$ operate horizontally. We can use the saturation condition SSdc in order to add new points and eliminate such counterexamples. As an example, consider the case when a point $v_{1}$ has been inserted between two points $v_{0}$ and $v_{2}$ such that $v_{0} \simeq v_{0}^{\prime}$ and $v_{2} \simeq v_{2}^{\prime}$. The resulting defect can be cured by adding a point $v_{1}^{\prime}$ between $v_{0}^{\prime}$ and $v_{2}^{\prime}$ such that $v_{1} \simeq v_{1}^{\prime}$. Condition Ssdc ensures that a $v_{1}^{\prime}$ with the proper labeling exists. In both the cases (ii) and (iii), we deal with simple Wdc-defects and the frame can be extended by using condition SWdc, as described in point $(a)$ above.

In the following, when not working with the full language, we will anyway often require that the labeling set of formulas on which mosaics are defined is closed with respect to some properties. We will let such closure properties depend on the particular class of linear orders and the particular target branching class considered.

Definition 17. Let $\mathcal{C}$ be a class of linear orders and $\mathcal{D}$ a target branching class. $A$ set $\Lambda$ of formulas is said to be $\mathcal{C}$-D-closed if the following conditions are satisfied:

(i) $\Lambda$ is closed under subformulas and single negations (of non-negated formulas);

(ii) if Fst is in $\mathcal{C}$, then $(\mathrm{PH} \perp) \vee(\mathrm{H} \perp) \in \Lambda$.

(iii) if Lst is in $\mathcal{C}$, then $(\mathrm{FG} \perp) \vee(\mathrm{G} \perp) \in \Lambda$;

(iv) if $N$ st is in $\mathcal{C}$, then $\mathrm{P} \top \in \Lambda$;

(v) if Nlst is in $\mathcal{C}$, then $\mathrm{F} \top \in \Lambda$;

(vi) if $M b^{-}$is in $\mathcal{D}$, then $\mathrm{G} \perp \in \Lambda$.

Now we can use the procedure described in Lemma 2 to build, via an $\omega$ construction, a structure of the given type. The following result from [31] will be useful in order to ensure that relational properties are preserved during the construction.

Lemma 3. Let $\mathcal{D}$ be a target branching class and $F=\left\{F_{\lambda} \mid \lambda<\mu\right\}$ a set of ()-D-frames indexed on the ordinal $\mu$ such that $F_{\lambda} \subseteq F_{\lambda^{\prime}}$ for all $\lambda<\lambda^{\prime}<\mu$. Then the union $F=\bigcup_{\lambda<\mu} F_{\lambda}$ is a ()-D-frame. 
Theorem 1. Let $\mathcal{C}$ be a class of linear orders with $\mathcal{C}$ not including any of Udsc and Ddsc, $\mathcal{D}$ a target branching class and $\Gamma$ a set of formulas. Then, $\Gamma$ is $\mathcal{C}-\mathcal{D}$ satisfiable iff there exists a $\mathcal{C}$-D-structure of mosaics for $\Gamma$.

Proof. $(\Rightarrow)$ Let $M=(\mathcal{W}, \prec, \simeq, \mathcal{V})$ be a $\mathcal{C}$ - D-structure satisfying $\Gamma$ and let $u \in \mathcal{W}$ be a point such that $M, u \models \Gamma$. Given a set $\Lambda^{\prime}$, which contains $\Gamma$ and is $\mathcal{C}$ $\mathcal{D}$-closed, we can associate a different fresh atom, i.e., an atom that is not in $\Lambda^{\prime}$, to each world in $\mathcal{W}^{6}$. Let $\Lambda^{\prime \prime}$ be the smallest $\mathcal{C}$ - $\mathcal{D}$-closed set of formulas containing such atoms and $\Lambda=\Lambda^{\prime} \cup \Lambda^{\prime \prime}$. We associate a subset of $\Lambda$ to every point of $\mathcal{W}$ as follows: for every $v \in \mathcal{W}$ we define $\Delta_{v}=\left\{A \in \Lambda^{\prime} \mid M, v \models\right.$ $A\} \cup\left\{p_{v}\right\} \cup\left\{\neg p \mid p \in \Lambda^{\prime \prime}\right.$ and $\left.p \neq p_{v}\right\}$, where $p_{v}$ is the atom associated to $v$. Then we define the set $S_{V}=\left\{\left(\Delta_{v}, \Delta_{v^{\prime}}\right) \mid v, v^{\prime} \in \mathcal{W}\right.$ and $\left.v \prec v^{\prime}\right\} \cup\left\{\left(\Delta_{v}\right) \mid\right.$ $v \in \mathcal{W}$ and for all $v^{\prime} \in \mathcal{W}$ we have $v \nprec v^{\prime}$ and $\left.v^{\prime} \nprec v\right\}$. Similarly, we define $S_{H}=\left\{\left(\Delta_{v}, \Delta_{v^{\prime}}\right) \mid v, v^{\prime} \in \mathcal{W}\right.$ and $\left.v \simeq v^{\prime}\right\}$. It is easy to verify that $S=\left(S_{V}, S_{H}\right)$ is a $\mathcal{C}$-D -structure of mosaics. In fact, coherence and saturation conditions are clearly satisfied since the definition of each point in $S$ comes from the labeling of the corresponding point in a $\mathcal{C}$ - $\mathcal{D}$-structure and the use of fresh atoms ensures that each world in $\mathcal{W}$ gives rise to a distinct point in $S$. Furthermore $S$ is a structure of mosaics for $\Gamma$ since $\Gamma \subseteq \Delta_{u}$ and $\Delta_{u} \in \operatorname{Points}(S)$. $(\Leftarrow)$ Let $S$ be a $\mathcal{C}$-D -structure of mosaics for $\Gamma$ on a $\mathcal{C}$ - $\mathcal{D}$-closed labeling set $\Lambda$ of formulas. As in [16], we build a model for $\Gamma$ step by step by using the mosaics in $S$ as building blocks. The procedure described in Lemma 2 will be used to cure the defects. Namely, we define a sequence $\sigma$ containing all the formulas FA, P $A, \exists A$ in $\Lambda$ such that each such formula occurs infinitely often in $\sigma$ and proceed as follows.

Notice that we cannot guarantee that the result of each step of the construction is a $\mathcal{C}$ - $\mathcal{D}$-chronicled frame because some of the properties (both linear and branching) will only emerge in the limit step. Namely, during the intermediate steps of the construction we will work with ()- $\mathcal{D}$-chronicled frames, for $\mathcal{D}=()$, $\mathcal{D}=(\mathrm{Wdc})$ and $\mathcal{D}=(\mathrm{Wdc}+\mathrm{Sdc})$, and with ()$-(W d c+S d c)$-chronicled frames if $\mathcal{D}=\left(\mathrm{Wdc}+\mathrm{Sdc}+\mathrm{Mb}^{-}\right)$.

[STEP 0] First, let us consider a mosaic $\mu$ in $S$ such that $\mu$ is a mosaic for $\Gamma$ (since $S$ is a structure of mosaics for $\Gamma$, such a mosaic exists). Moreover, since by definition Points $\left(S_{V}\right)=\operatorname{Points}\left(S_{H}\right)$, we can assume, without loss of generality, that $\mu$ is a vertical mosaic. We can define $F_{0}=\left\{\mathcal{W}_{0}, \prec_{0}, \simeq_{0}\right\}$ as follows. If $\mu=\left(\Delta_{0}\right)$ is a singleton, then we define $\mathcal{W}_{0}=\left\{w_{0}\right\}, \prec_{0}=\emptyset$ and $\simeq_{0}=\left\{w_{0}, w_{0}\right\}$. Furthermore, we associate to $\mathcal{W}_{0}$ the chronicle $\delta_{0}$ defined as $\delta_{0}\left(w_{0}\right)=\Delta_{0}$. If $\mu=\left(\Delta_{0}, \Delta_{1}\right)$ is a vertical mosaic in $S_{V}$, then we define $\mathcal{W}_{0}=\left\{w_{0}, w_{1}\right\}, \prec_{0}=$ $\left\{\left(w_{0}, w_{1}\right)\right\}$ and $\simeq_{0}=\left\{\left(w_{0}, w_{0}\right),\left(w_{1}, w_{1}\right)\right\}$. We associate to $\mathcal{W}_{0}$ the chronicle $\delta_{0}$ defined as $\delta_{0}\left(w_{0}\right)=\Delta_{0}$ and $\delta_{0}\left(w_{1}\right)=\Delta_{1}$. In both cases $F_{0}$ is a ()-D -frame and $\delta_{0}$ is a chronicle for $F_{0}$ based on $S$.

$[S T E P \quad n+1]$ Assume that we have already defined a ()-D -frame (a ()-( $W d c+$ $S d c)$-frame if $\left.\mathcal{D}=\left(\mathrm{Wdc}+\mathrm{Sdc}+\mathrm{Mb}^{-}\right)\right) F_{n}$ and a chronicle $\delta_{n}$ for $F_{n}$ based on $S$. Then we consider the $(n+1)$-th formula $A$ in the enumeration $\sigma$. By using

\footnotetext{
${ }^{6}$ By adapting the result from the Löwenheim-Skolem theorem (see, e.g., [28]), we can assume, without loss of generality, that $\mathcal{W}$ is countable.
} 
the procedure described in Lemma 2, we can define a ()- $\mathcal{D}$-frame $F_{n+1}$ and a chronicle $\delta_{n+1}$ for it such that for each defect $\langle w, A\rangle$ in $\left(F_{n}, \delta_{n}\right)$, we have that it is not a defect in $\left(F_{n+1}, \delta_{n+1}\right)$.

Notice that if $\mathcal{D}=\left(\mathrm{Wdc}+\mathrm{Sdc}+\mathrm{Mb}^{-}\right)$, we just apply the procedure described for $\mathcal{D}=(W d c+S d c)$. Moreover, in some cases, depending on the nature of $\mathcal{C}$, we slightly refine the procedure described in Lemma 2. Namely, if $\mathcal{C}$ contains Dns, then, as suggested in [16], when curing vertical defects we add not only the mosaics specified by the procedure of Lemma 2 but also, between all the neighboring points, all the mosaics that can lay in the middle. Condition SVDns ensures that there is at least one such mosaic for each pair of neighboring points.

$[S T E P \omega]$ Now we can just take the infinite unions $F=\bigcup_{i \in \omega} F_{i}$ and $\delta=$ $\bigcup_{i \in \omega} \delta_{i}$. The special curing procedure described above for the dense case guarantees that the process will finally produce a frame which is dense. In the case of the other linear properties possibly contained in $\mathcal{C}$, it is the definition of mosaic itself to guarantee that the property is enjoyed by the frame obtained in the limit step.

Then, by Lemma $3, F$ is a $\mathcal{C}$ - $\mathcal{D}$-frame, for $\mathcal{D}=(), \mathcal{D}=(W d c)$ or $\mathcal{D}=$ $(W d c+S d c)$. In the case when $\mathcal{D}=\left(\mathrm{Wdc}+\mathrm{Sdc}+\mathrm{Mb}^{-}\right)$, in the intermediate steps of the construction we have frames (with associated chronicles) which enjoy $\mathrm{Wdc}$ and Sdc but not necessarily $\mathrm{Mb}^{-}$. However, condition $\mathrm{SMb}^{-}$ensures that, at each step $i$, a $\prec$-maximal point $w$ can be $\simeq$-related to a point $v$ distinct from $w$ only if $\mathrm{G} \perp \notin \delta_{i}(w)$. But this implies that $w$ contains a vertical future defect, which in some later step will be cured by inserting some point above $w$. Thus in the final construction we have that also $\mathrm{Mb}^{-}$is satisfied. Furthermore, in all the cases, by the construction we have no defects in $(F, \delta)$, since the enumeration in $\sigma$ ensures that if a defect becomes actual at some step, then we cure it in a later step.

We can easily obtain a $\mathcal{C}$ - $\mathcal{D}$-structure by endowing $F$ with a valuation $\mathcal{V}$ induced by $\delta$. Namely, let $F$ be $(\mathcal{W}, \prec, \simeq)$; then we define a structure $M=$ $(\mathcal{W}, \prec, \simeq, \mathcal{V})$, where $\mathcal{V}$ is such that for all $u \in \mathcal{W}$ and for all atomic propositions $p, p \in \mathcal{V}(u)$ iff $p \in \delta(u)$. By recalling that we used a mosaic for $\Gamma$ as a foundation stone of our construction (STEP 0), we conclude that $M$ is a $\mathcal{C}$ - $\mathcal{D}$-structure that satisfies $\Gamma$.

Corollary 1. Let $\mathcal{C}$ be a class of linear orders with $\mathcal{C}$ not including any of Udsc and Ddsc, $\mathcal{D}$ a target branching class and $\Gamma$ a set of formulas. Then $\Gamma$ is $\mathcal{C}$ $(D i s+W d c)$-satisfiable iff there exists a $\mathcal{C}-(W d c+S d c)$-structure of mosaics for $\Gamma . \Gamma$ is $\mathcal{C}$-Ockhamist-satisfiable (i.e., by Lemma 1, satisfiable in the logic defined over bundled trees in the class $\left.\mathcal{B}^{+}(\mathcal{C})[12]\right)$ iff there exists a $\mathcal{C}-\left(W d c+S d c+M b^{-}\right)$structure of mosaics for $\Gamma$.

Proof. This follows straightforwardly by combining the result of Theorem 1 and the equivalences of Lemma 1.

In the case where no interactions between the components are considered, i.e., when $\mathcal{D}=()$, it is possible to give a proof based on a labeling set which is 
finite. This observation will be crucial in obtaining a result of decidability (see Section 4.3 below). We remark that in this case we are able to deal also with discreteness properties.

Theorem 2. Let $\mathcal{C}$ be a class of linear orders and $\Gamma$ a finite set of formulas. Then, $\Gamma$ is $\mathcal{C}$-()-satisfiable iff there exists a $\mathcal{C}$-()-structure of mosaics for $\Gamma$ on a finite labeling set.

Proof. $(\Rightarrow)$ Since $\Gamma$ is $\mathcal{C}$-()-satisfiable, there exist $M=(\mathcal{W}, \prec, \simeq, \mathcal{V})$ and $u \in \mathcal{W}$ such that $M, u \models \Gamma$. Let $\Lambda$ be the smallest $\mathcal{C}$-()-closed set of formulas containing $\Gamma$. We will show that there exists a structure of mosaics on $\Lambda$ for $\Gamma$.

We can easily infer a set of mosaics on the labeling set $\Lambda$ from $M$. We associate a subset of $\Lambda$ to every point of $\mathcal{W}$ as follows: first we define $\Delta_{w}=\{A \in$ $\Lambda \mid M, w \models A\}$ for every $w \in \mathcal{W}$. Then we define the sets $S_{V}=\left\{\left(\Delta_{w}, \Delta_{w}^{\prime}\right) \mid\right.$ $w, w^{\prime} \in \mathcal{W}$ and $\left.w \prec w^{\prime}\right\} \cup\left\{\left(\Delta_{w}\right) \mid w \in \mathcal{W}\right\}$ and $S_{H}=\left\{\left(\Delta_{w}, \Delta_{w}^{\prime}\right) \mid w, w^{\prime} \in\right.$ $\mathcal{W}$ and $\left.w \simeq w^{\prime}\right\} \cup\left\{\left(\Delta_{w}\right) \mid w \in \mathcal{W}\right\}$. It is easy to verify that $S=\left(S_{V}, S_{H}\right)$ is indeed a $\mathcal{C}$-()-structure of mosaics. In fact coherence and saturation conditions are clearly satisfied since the definition of each point in $S$ comes from the labeling of the corresponding point in a $\mathcal{C}$-()-structure. Furthermore $S$ is a structure of mosaics for $\Gamma$, since $\Gamma \subseteq \Delta_{u}$ and $\Delta_{u} \in \operatorname{Points}(S)$.

$(\Leftarrow)$ The thesis follows from a construction analogous to that in the proof of Theorem 1 (right-to-left direction). Clearly, restricting to consider structures of mosaics based on a finite labeling set does not affect the previous result.

In this case, we can also consider classes of linear orders satisfying Ddsc and/or Udsc. Namely, if $\mathcal{C}$ contains UDsc, then in cases like $(b)$ for the curing of vertical future mosaics (Lemma 2), we proceed by adding a point $v^{*}$ between $v^{\prime}$ and $v^{\prime \prime}$ such that $\{A, \neg \mathrm{F} A\} \subseteq \delta^{\prime}\left(v^{*}\right)$. Condition SVUdsc ensures that there exists a mosaic allowing that. If $\mathcal{C}$ contains DDsc, then we proceed symmetrically in the case of vertical past defects. The fact that the labeling set is finite guarantees that only a finite number of formulas of the form $\mathrm{F} A$ or $\mathrm{P} A$ occurs in any point. Hence, between any two points, we will insert only finitely many points during our $\omega$-construction.

\section{Applications}

In this section, we study some applications of the mosaic method defined above. In particular, we describe a mosaic-based proof of completeness for a Hilbertstyle axiomatization for our Ockhamist branching temporal logics, we formulate and study mosaic-based tableaux systems, and study the decidability of some of these logics.

\subsection{Hilbert-style completeness via mosaics}

One of the possible applications of the mosaic method is in proving the completeness of a given deduction system (as, e.g., in $[21,16]$ ). In fact, Theorem 1 can be used to simplify the standard proof of completeness: given a consistent 
set of formulas we do not need to create a model satisfying it; a structure of mosaics will suffice.

A standard ([31]) proof of completeness for axiomatic systems capturing the logics presented here consists in taking maximal consistent sets and defining two relations $\prec^{M}$ and $\simeq^{M}$ on them, based on the formulas they contain, i.e.,

$$
\Gamma \prec^{M} \Delta \text { iff }\{A \mid \mathrm{G} A \in \Gamma\} \subseteq \Delta \quad \text { and } \quad \Gamma \simeq^{M} \Delta \text { iff }\{A \mid \forall A \in \Gamma\} \subseteq \Delta .
$$

The idea is that such relations can be used as the basis for building a structure by a procedure of elimination of counterexamples [2,3,31]. If we use mosaics, then part of this procedure is already contained in the assert of Theorem 1 and it suffices to show that the structure $\left(S_{V}, S_{H}\right)$ is a structure of mosaics of the given class, where $S_{V}$ is the set of all pairs $(\Gamma, \Delta)$ of maximal consistent sets such that $\Gamma \prec^{M} \Delta$ and $S_{H}$ is the set of all pairs $(\Gamma, \Delta)$ of maximal consistent sets such that $\Gamma \simeq^{M} \Delta$.

Hilbert-style axiomatizations Here we list some axioms and rules of inference that will give rise to a hierarchy of Hilbert-style axiomatizations for the logics considered. Here we will consider strong completeness and, as in Theorem 1, drop the discreteness conditions Udsc and Ddsc. We remark that, by exploiting the result of Theorem 2, a proof of weak completeness would be possible for axiomatizations capturing also discreteness but in a setting with no interactions between the components, i.e., for $\mathcal{D}=()$.

Vertical axioms (for general linear-time)

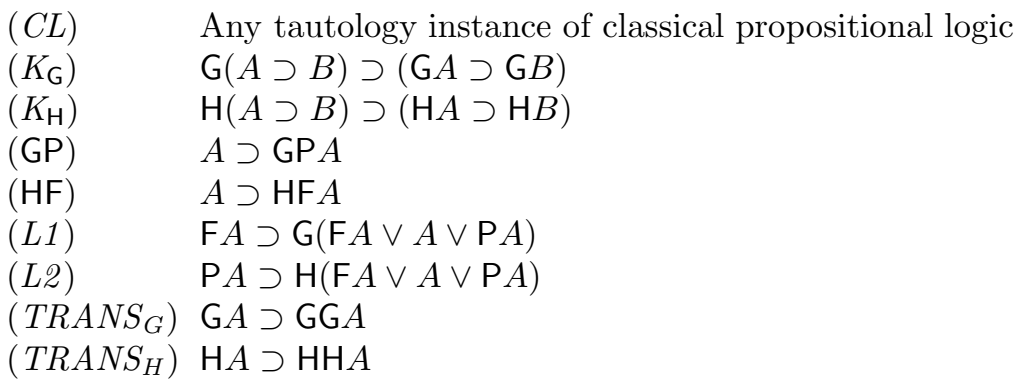

Axioms for particular linear flows

$$
\begin{array}{ll}
(\text { Fst } A) & \mathrm{H} \perp \vee \mathrm{PH} \perp \\
(\text { Lst } A) & \mathrm{G} \perp \vee \mathrm{FG} \perp \\
(\text { Nfst } A) & \mathrm{P} \top \\
(\text { Nlst } A) & \mathrm{F} \top \\
(\text { Dns } A) & \mathrm{F} A \supset \mathrm{FF} A \wedge \mathrm{P} A \supset \mathrm{PP} A
\end{array}
$$

Horizontal axioms ( $S 5$ with respect to the operator $\forall$ )

$$
\begin{aligned}
& \left(K_{\forall}\right) \forall(A \supset B) \supset(\forall A \supset \forall B) \\
& (\forall 1) \quad \forall A \supset \forall \forall A \\
& (\forall \mathscr{2}) \quad \forall A \supset A \\
& (\forall 3) \quad A \supset \forall \exists A
\end{aligned}
$$


Branching axioms (compositional and harmonic properties)

$$
\begin{array}{ll}
(W d c A) & \mathrm{P} A \supset \forall \mathrm{P} \exists A \\
\left(M b^{-} A\right) & \mathrm{G} \perp \wedge \exists A \supset A \\
(A t m A) & p \supset \forall p, \text { for } p \in \mathcal{P} .
\end{array}
$$

Rules of deduction

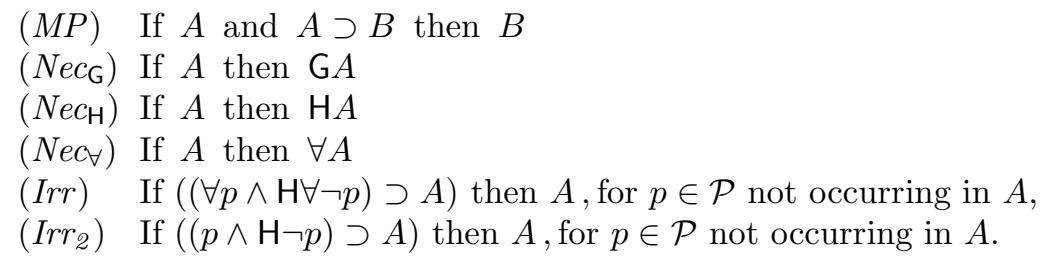

For each of our $\mathcal{L}(\mathcal{C}, \mathcal{D})$ logics of interest, such that Udsc and Dsc are not in $\mathcal{C}$, we can obtain an axiomatization $\mathcal{H}(\mathcal{C}, \mathcal{D})$ by including:

- the vertical axioms for general linear time

$\left(C L, K_{G}, K_{H}, G P, H F, L 1, L 2, T R A N S_{G}, T R A N S_{H}\right)$, the horizontal axioms $\left(K_{\forall}, \forall 1, \forall 2, \forall 3\right)$, and the necessitation rules $\left(\mathrm{Nec}_{G}, \mathrm{Nec} \mathrm{c}_{H}, \mathrm{Nec} \forall\right)$;

- axioms for particular linear flows

$(C A)$, for each condition $C$ listed in $\mathcal{C}$;

- additional axioms and rules for particular target branching classes

- $\mathcal{D}=(\mathrm{Wdc})$

$(W d c A)$;

- $\mathcal{D}=(\mathrm{Wdc}+\mathrm{Sdc})$

$(W d c A, \operatorname{Irr})^{7}$

- $\mathcal{D}=\left(\mathrm{Wdc}+\mathrm{Sdc}+\mathrm{Mb}^{-}\right)$

( $\left.W d c A, \operatorname{Irr}, M^{-} A\right)$.

In the case when the atomic harmony assumption is satisfied (see Section 2), a proper axiomatization can be obtained [31] by adding the axiom (AtmA). Moreover, the rule ( $\mathrm{Irr}$ ) can be reformulated as $\left(\operatorname{Irr}_{2}\right)$.

\footnotetext{
${ }^{7}$ As an alternative formulation, we notice that in [30], Zanardo proposes the following two rather complex (but with a standard form) Hilbert-style axioms:

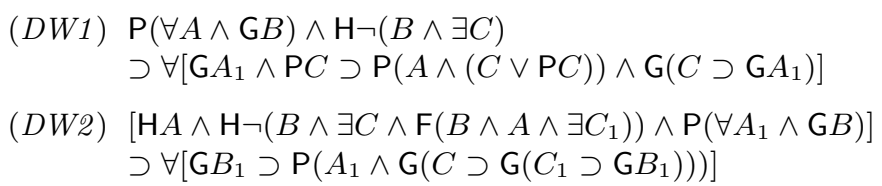

The addition of such axioms to $\mathcal{H}(\mathcal{C},(W d c))$ gives rise to an axiomatization for the $\operatorname{logic} \mathcal{L}(\mathcal{C},(W d c+S d c))$; see [30] for a proof.
} 
Given a class $\mathcal{C}$ of linear orders, and a target branching class $\mathcal{D}$, the notions of $\mathcal{H}(\mathcal{C}, \mathcal{D})$-theoremhood, $\mathcal{H}(\mathcal{C}, \mathcal{D})$-derivability and $\mathcal{H}(\mathcal{C}, \mathcal{D})$-consistency are defined in the standard way. A set $\Gamma$ of formulas is further said to be maximally $\mathcal{H}(\mathcal{C}, \mathcal{D})$ consistent if either $A \in \Gamma$ or $\neg A \in \Gamma$ for every $A \in \mathcal{F}$. If $\Gamma$ is not $\mathcal{H}(\mathcal{C}, \mathcal{D})$ consistent, it will be called $\mathcal{H}(\mathcal{C}, \mathcal{D})$-inconsistent.

Completeness via mosaics In the rest of this section, for $\mathcal{C}$ a class of linear orders and $\mathcal{D}$ a target branching class, we will denote with $M C_{\mathcal{C}, \mathcal{D}}$ the set of maximal consistent sets of formulas generated by the axiomatization $\mathcal{H}(\mathcal{C}, \mathcal{D})$. We will prove completeness of the given axiomatizations by using mosaics and by adapting known results, mainly from [31] and [7], where axiomatizations very close to ours are presented.

Theorem 3. Let $\Omega$ be a set of formulas and $\mathcal{C}$ a class of linear orders such that $U d s c$ and Ddsc are not in $\mathcal{C}$. If $\Omega$ is $\mathcal{H}(\mathcal{C},())$-consistent, then there exists a $\mathcal{C}$-()-structure of mosaics for $\Omega$.

Proof. We have to show that there is a $\mathcal{C}$-()-structure of mosaics for $\Omega$. Let our labeling set be the set of all formulas. Then we define the set $S_{V}$ as follows:

$$
\begin{aligned}
& S_{V}=\left\{(\Gamma) \mid \Gamma \in M C_{\mathcal{C},()}\right\} \cup \\
& \left.\qquad(\Gamma, \Delta) \mid \Gamma, \Delta \in M C_{\mathcal{C},()} \text { and }\{A \mid \mathrm{G} A \in \Gamma\} \subseteq \Delta\right\}
\end{aligned}
$$

It is easy to prove that the following definitions are equivalent to (1).

$$
\begin{gathered}
S_{V}=\left\{(\Gamma) \mid \Gamma \in M C_{\mathcal{C},()}\right\} \cup \\
\left\{(\Gamma, \Delta) \mid \Gamma, \Delta \in M C_{\mathcal{C},()} \text { and }\{A \mid \mathrm{H} A \in \Delta\} \subseteq \Gamma\right\} \\
S_{V}=\left\{(\Gamma) \mid \Gamma \in M C_{\mathcal{C},()\}} \cup\right. \\
\left\{(\Gamma, \Delta) \mid \Gamma, \Delta \in M C_{\mathcal{C},()} \text { and }\{\mathrm{F} A \mid A \in \Delta\} \subseteq \Gamma\right\} \\
S_{V}=\left\{(\Gamma) \mid \Gamma \in M C_{\mathcal{C},()}\right\} \cup \\
\left\{(\Gamma, \Delta) \mid \Gamma, \Delta \in M C_{\mathcal{C},()} \text { and }\{\mathrm{P} A \mid A \in \Gamma\} \subseteq \Delta\right\}
\end{gathered}
$$

Similarly we can define $S_{H}$ as:

$$
\begin{aligned}
S_{H}=\left\{(\Gamma) \mid \Gamma \in M C_{\mathcal{C},()}\right\} \cup \\
\left\{(\Gamma, \Delta) \mid \Gamma, \Delta \in M C_{\mathcal{C},()} \text { and }\{A \mid \forall A \in \Gamma\} \subseteq \Delta\right\}
\end{aligned}
$$

which can be proven to be equivalent to:

$$
\begin{aligned}
& S_{H}=\left\{(\Gamma) \mid \Gamma \in M C_{\mathcal{C},()}\right\} \cup \\
& \left\{(\Gamma, \Delta) \mid \Gamma, \Delta \in M C_{\mathcal{C},()} \text { and }\{\exists A \mid A \in \Delta\} \subseteq \Gamma\right\}
\end{aligned}
$$

Now we define $S=\left(S_{V}, S_{H}\right)$ and claim that $S$ is indeed a $\mathcal{C}$-()-structure of mosaics. By Definition 14, we need to prove that: 
(i) $S_{V}$ is a $\mathcal{C}$-VSSM;

(ii) $S_{H}$ is an HSSM;

(iii) $M \in \operatorname{Points}(S)$ implies $M \in \operatorname{Points}\left(S_{V}\right)$ and $M \in \operatorname{Points}\left(S_{H}\right)$.

The proof uses the same arguments in the proof of completeness of [31]. Firstly, by using classical tautologies and $(\forall 2)$, we can show that each $\Gamma \in$ Points $(S)$ is a point. In particular, in the case of $\mathcal{C}$ containing Fst, Lst, Nfst or Nlst, the corresponding axiom directly ensures that each point is a PB-point, an FB-point, a PU-point or an FU-point, respectively.

Moreover, by (1) and $\left(T R A N S_{G}\right)$, each element of $S_{V}$ is a vertical mosaic and, by (5) and $(\forall 2)$ (and $(A H)$ if atomic harmony is assumed), each element of $S_{H}$ is a horizontal mosaic.

Also, by standard Kripke-style methods (see [31]), one can show that if $\mathrm{F} A(\mathrm{P} A, \exists A) \in \Gamma$, then there exists a $\Delta$ such that $A \in \Delta$ and $(\Gamma, \Delta) \in S_{V}$ $\left((\Delta, \Gamma) \in S_{V},(\Gamma, \Delta) \in S_{H}\right)$. This implies that $S_{V}$ is a ()-VSSM and $S_{H}$ is an HSSM. With regard to point (i), when $\mathcal{C}$ is some particular class of linear orders, we can refine this result by observing that:

- in the case of $\mathcal{C}$ containing Fst (Lst, Nfst or Nlst), we have automatically that $S_{V}$ is a set of $\mathrm{PB}(\mathrm{FB}, \mathrm{PU}$, or FU, respectively)-mosaics and thus a $\mathcal{C}$-VSSM;

- in the case when $\mathcal{C}$ contains Dns, Udsc or Ddsc, the axioms $(D n s A),(U d s c A)$ or $(D d s c A)$ can be used to prove the corresponding saturation properties on $S$, by using the standard techniques of, e.g., [1].

Finally, as for (iii), we observe that, by (1) and (5), Points $\left(S_{V}\right)=M C_{\mathcal{C},()}=$ Points $\left(S_{H}\right)$.

By considering the standard result [31] according to which every consistent set of formulas is contained in a maximal consistent set of formulas, we have that there exists a $\Omega^{\prime}$ such that $\Omega \subseteq \Omega^{\prime}$ and $\Omega^{\prime} \in \operatorname{Points}(S)$ and thus that $S$ is a structure of mosaics for $\Omega$.

Theorem 4. Let $\Omega$ be a set of formulas and $\mathcal{C}$ a class of linear orders such that $U d s c$ and $D d s c$ are not in $\mathcal{C}$. If $\Omega$ is $\mathcal{H}(\mathcal{C},(W d c))$-consistent, then there exists a $\mathcal{C}$ - $(W d c)$-structure of mosaics for $\Omega$.

Proof. We can define a structure of mosaics $S$ as in Theorem 3. Then, by using the axiom $(W d c A)$ and standard compactness techniques [31], we have that $S$ enjoys SWdc. Moreover, as proven in [31], the set $S_{V}$ enjoys transitivity (STrn) and connectedness $(\mathrm{SCon})$ and is therefore a $\mathcal{C}$ - $(\mathrm{Wdc})$-structure of mosaics.

In the case of $(\mathrm{Wdc}+\mathrm{Sdc})$-structures, we refer to an axiomatization enriched by the rule (Irr) (see above), which forces the construction of irreflexive models $[6,7]$. Namely, such a rule allows for giving each point a unique "name", in the sense that each point is characterized by the fact of being the $\prec$-minimal point where a certain atom holds.

We can define a structure of mosaics in a way very similar to that of Theorems 3 and 4 . However, we will now restrict our attention to a subset of maximal 
consistent sets, the so-called Irr-sets (see also [7] for an illustration of their use in proving completeness).

Definition 18. A set $\Gamma$ of formulas is an Irr-set iff for all $n \geq 0$, for all $\diamond_{1}, \ldots, \nabla_{n} \in\{\mathrm{F}, \mathrm{P}, \exists\}$, for all formulas $A_{0}, \ldots, A_{n}$, if

$$
A_{0} \wedge \nabla_{1}\left(A_{1} \wedge \nabla_{2}\left(A_{2} \wedge \ldots \nabla_{n}\left(A_{n}\right) \ldots\right)\right) \in \Gamma
$$

then there is a propositional variable $p \in \mathcal{P}$ such that

$$
A_{0} \wedge \diamond_{1}\left(A_{1} \wedge \diamond_{2}\left(A_{2} \wedge \ldots \diamond_{n}\left(A_{n} \wedge \exists p \wedge \mathrm{H} \neg \exists p\right) \ldots\right)\right) \in \Gamma
$$

Definition 19. Let $\mathcal{C}$ be a class of linear orders. We define the set $I M C_{\mathcal{C}},(W d c+S d c)$

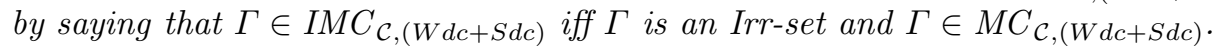

Theorem 5. Let $\Omega$ be a set of formulas and $\mathcal{C}$ a class of linear orders such that $U d s c$ and Ddsc are not in $\mathcal{C}$. If $\Omega$ is $\mathcal{H}(\mathcal{C},(W d c+S d c))$-consistent, then there exists a $\mathcal{C}-(W d c+S d c)$-structure of mosaics for $\Omega$.

Proof. As in Theorems 3 and 4, we will build a structure of mosaics and show that it is indeed a $\mathcal{C}-(\mathrm{Wdc}+\mathrm{Sdc})$-structure of mosaics for $\Omega$.

Firstly, we notice that if $\Omega$ is consistent then it can be extended to a set

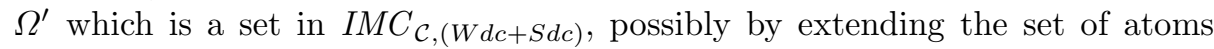
considered (see, e.g., [7] for a proof of this fact). Let our labeling set be the set of all formulas in such a (possibly extended) set of atoms. Then, similarly to the proof of Theorem 3, we define the set $S_{V}$ as follows:

$$
\begin{aligned}
& S_{V}=\left\{(\Gamma) \mid \Gamma \in I M C_{\mathcal{C},(W d c+S d c)}\right\} \cup \\
&\left\{(\Gamma, \Delta) \mid \Gamma, \Delta \in I M C_{\mathcal{C},(W d c+S d c)} \text { and }\{A \mid \mathrm{G} A \in \Gamma\} \subseteq \Delta\right\}
\end{aligned}
$$

and the set $S_{H}$ as follows:

$$
\begin{aligned}
& S_{H}=\left\{(\Gamma) \mid \Gamma \in I M C_{\mathcal{C},(W d c+S d c)}\right\} \cup \\
&\left\{(\Gamma, \Delta) \mid \Gamma, \Delta \in I M C_{\mathcal{C},(W d c+S d c)} \text { and }\{A \mid \forall A \in \Gamma\} \subseteq \Delta\right\}
\end{aligned}
$$

As in the proof of Theorem 3, equivalent definitions are possible (we omit them). Now we claim that $S=\left(S_{V}, S_{H}\right)$ is a $\mathcal{C}$ - $(\mathrm{Wdc}+\mathrm{Sdc})$-structure of mosaics for $\Omega$.

First of all, we notice that it is not difficult to extend the results of Theorems 3 and 4 to this case. In particular, we refer the reader to the treatment in [7]: vertical and horizontal saturation conditions are satisfied as a direct consequence of [7, Lemma 7.7.6] and SWdc holds because of [7, Lemma 7.7.9].

It remains to show that $S$ enjoys SSdc. We know (as a direct consequence of Lemma 7.7.7 in [7]) that $S$ enjoys a variant of the property (Dsj) (as defined in Section 2), i.e., if $(\Gamma, \Delta) \in S_{V}$ and $\left(\Delta, \Delta^{\prime}\right) \in S_{H}$ then $\left(\Gamma, \Delta^{\prime}\right) \notin S_{H}$. Moreover, as proved in Lemma 7.7.9 of [7], $S$ enjoys S-Wdc. By observing that the two properties imply S-Sdc, we have the assert.

This shows that $S$ is a $\mathcal{C}$ - $\left(\mathrm{Wdc}+\mathrm{Sdc}+\mathrm{Mb}^{-}\right)$-structure of mosaics. Since $\Omega \subseteq$ $\Omega^{\prime}$ and $\Omega^{\prime} \in I M C_{\mathcal{C},\left(W d c+S d c+M b^{-}\right)}, S$ is a structure of mosaics for $\Omega$. 


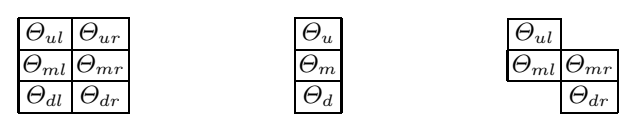

Fig. 2. Sample graphical representations of partial tuples.

Theorem 6. Let $\Omega$ be a set of formulas and $\mathcal{C}$ a class of linear orders such that $U d s c$ and Ddsc are not in $\mathcal{C}$. If $\Omega$ is $\mathcal{H}\left(\mathcal{C},\left(W d c+S d c+M b^{-}\right)\right)$-consistent, then there exists a $\mathcal{C}-\left(W d c+S d c+M b^{-}\right)$-structure of mosaics for $\Omega$.

Proof. We only need to prove that the structure $S$, defined as in the proof of Theorem 5 , but with respect to $I M C_{\mathcal{C},\left(W d c+S d c+M b^{-}\right)}$, also enjoys $\mathrm{SMb}^{-}$. By the sake of contradiction, assume there exist $\Gamma, \Delta$ such that $\mathrm{G} \perp \in \Gamma,(\Gamma, \Delta) \in S_{H}$ and $\Gamma \neq \Delta$. Then there exists $A \in \Delta$ such that $A \notin \Gamma$. By $(\Gamma, \Delta) \in S_{H}$ and $A \in \Delta$, it follows $\exists A \in \Gamma$. Then, by using the axiom $\left(M b^{-} A\right)$, we get $A \in \Gamma$ (absurd).

\subsection{Mosaic-based tableaux}

It is relatively simple to extract quite appealing semantic tableau systems for our logics directly from the mosaics definition. The syntactical elements of our tableaux systems are partial 6-tuples properly labelled with sets of formulas, plus the particle CLOSED that will stand for an absurd. Rigorously, a partial 6 -tuple is simply a partial function $\Theta:\{u l, u r, m l, m r, d l, d r\} \nrightarrow 2^{\mathcal{F}}$. The letters $d, m, u$ and $l, r$ used in naming the elements of the domain of $\Theta$, the positions, stand for down, middle, up, and left, right, respectively. We will often depict these partial tuples graphically as shown in Fig. 2, omitting the undefined entries from the graphical representation, and assuming that, whenever defined, $\Theta(p)=\Theta_{p}$ for $p \in\{u l, u r, m l, m r, d l, d r\}$. In Fig. 2, we also depict the general form of the (linear only) 3-tuples used in [16], as well as of the tuples with the upper-right and down-left entries undefined (for illustration purposes).

The semantics is simple. A structure $\mathcal{M}=(\mathcal{W}, \prec, \simeq, \mathcal{V})$ is said to satisfy a tuple $\Theta:\{u l, u r, m l, m r, d l, d r\} \nrightarrow 2^{\mathcal{F}}$, in which case $\Theta$ is said to be satisfiable, if there exists a function $\omega:\{u l, u r, m l, m r, d l, d r\} \nrightarrow \mathcal{W}$ such that the following conditions hold:

- for every $p \in\{u l, u r, m l, m r, d l, d r\}$,

- $\omega(p)$ is defined iff $\Theta(p)$ is defined;

- if $\omega(p)$ is defined then $\mathcal{M}, \omega(p) \models \Theta_{p}$;

- for every $h \in\{l, r\}$,

- if $\Theta(d h)$ and $\Theta(m h)$ are both defined then $\omega(d h) \prec \omega(m h)$,

- if $\Theta(d h)$ and $\Theta(u h)$ are both defined then $\omega(d h) \prec \omega(u h)$,

- if $\Theta(m h)$ and $\Theta(u h)$ are both defined then $\omega(m h) \prec \omega(u h)$;

- for every $v \in\{d, m, u\}$,

- if $\Theta(v l)$ and $\Theta(v r)$ are both defined then $\omega(v l) \simeq \omega(v r)$. 

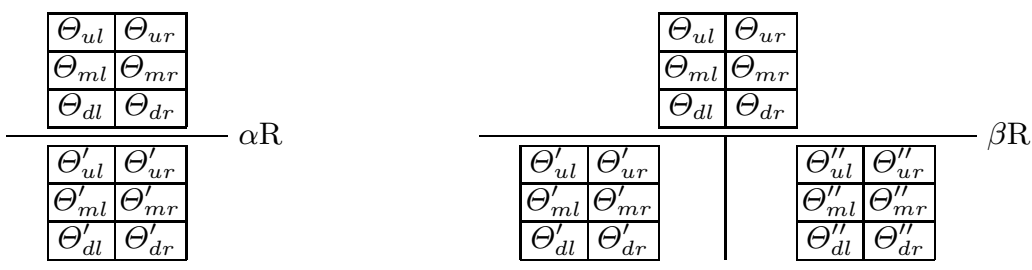

Fig. 3. General shape of tableau rules.

We also define the particle CLOSED to be unsatisfiable.

This explains well why we will work, in the general case, with such tuples: 3 vertically related points by 2 horizontally related points are what we need to be able to express all the mosaic conditions of the previous section (namely, the most complex one, that is strong diagram completion). Indeed, we can directly produce tableau rules that correspond to the mosaic conditions, either unary $(\alpha \mathrm{R})$, or binary $(\beta \mathrm{R})$ leading to a bifurcation in the tableau, as shown in Fig. 3.

The rules are given in Figures 4, 5, 6, 7 and 8. Dots represent context around the highlighted entries of the tuples that is meant to be preserved by the rules, but which can always be erased (neglected) using the deletion rule DelR in Fig. 4. In fact, the dots in the rule DelR represent the fact that any entry in a partial tuple can be deleted. Similarly, for instance, the dots in CutR represent the fact that we can apply a cut in any entry of a partial tuple, while there are rules, such as $\forall$ R2(left) in Fig. 5 or $\neg$ GR in Fig. 7, where the dots specify that only some of the context is meant to be preserved.

Fig. 4 also presents the basic propositional rules stemming (almost) immediately $^{8}$ from the definition of point in a mosaic structure, Definition 9, including in particular the unrestricted cut rule CutR and the closure rule ClsR.

Notice that a common rule for $\neg \neg$-elimination such as

\footnotetext{
${ }^{8}$ The immediate counterparts of condition (L2) in Definition 9 are obviously the rule $\wedge \mathrm{R}$ and a form of $\wedge$-introduction that could be expressed by a rule such as the one depicted below.
}

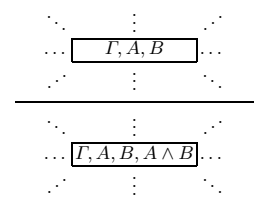

However, in the presence of CutR, it is not difficult to see that this rule turns out to be equivalent to the much more usual rule $\neg \wedge \mathrm{R}$, that we include. 


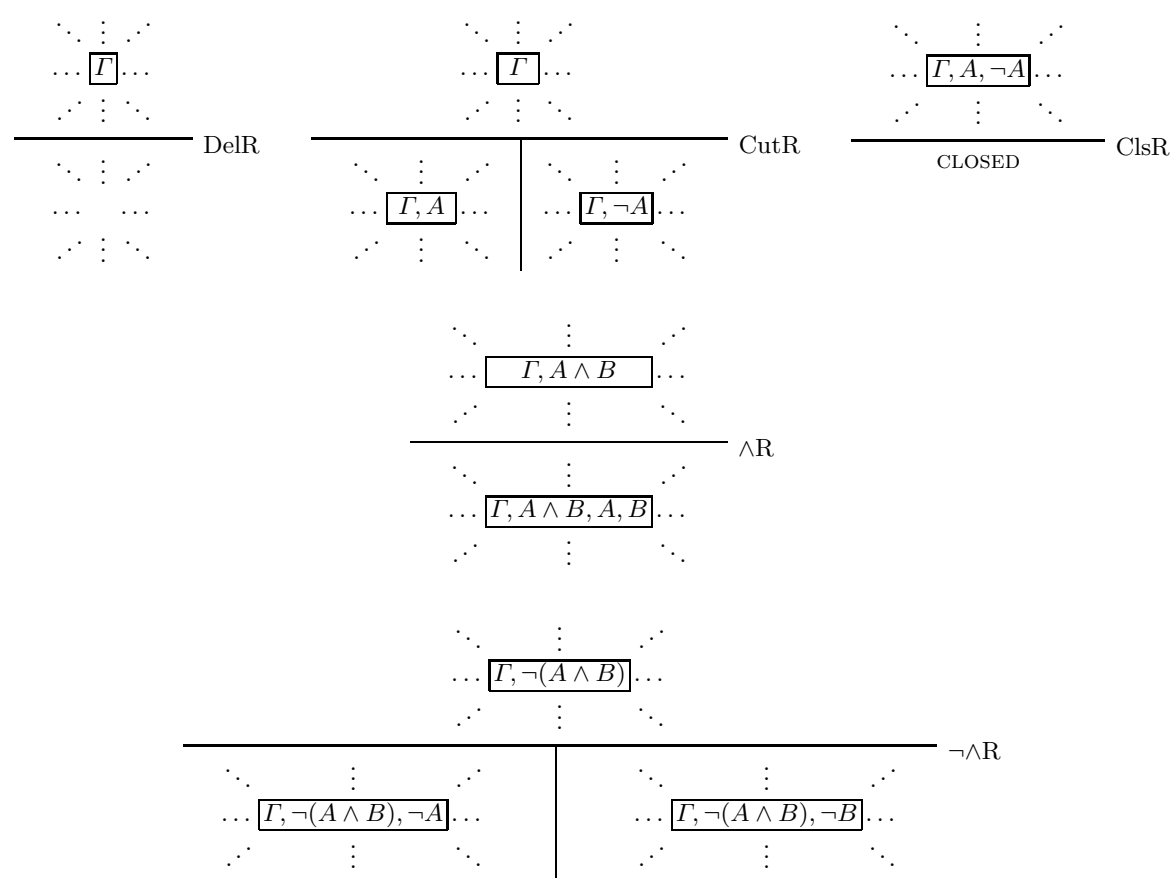

Fig. 4. Propositional and simplification rules.

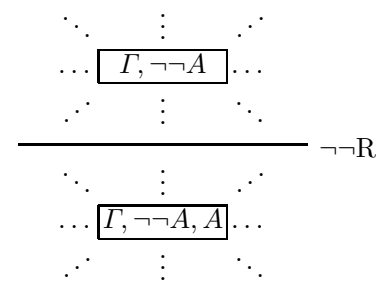

is not listed, since it is redundant given the (powerful) presence of CutR.

In Figure 5, we have the rules corresponding to the coherence conditions on mosaics, both for what concerns the vertical and the horizontal components. In Fig. 6, we find "special" coherence-based rules, namely AtmR rules capturing the atomic harmony assumption, rules NfstR and NlstR expressing unboundedness towards the past and towards the future, respectively, and rules $\mathrm{Mb}^{-} \mathrm{R}$ corresponding to the property of the maximality of branches. Rules corresponding to the saturation properties are presented in Fig. 7, where we have rules that mimic the curing of defects of Section 3.2, and in Fig. 8, where the special conditions on boundedness, discreteness and density of linear flows are captured together with rules that allow for representing the properties Wdc and Sdc.

We can now define a hierarchy of tableau systems $\mathcal{R}(\mathcal{C}, \mathcal{D})$ for each of our logics $\mathcal{L}(\mathcal{C}, \mathcal{D})$ (but with Udsc, Ddsc not in $\mathcal{C}$ ), by including: 


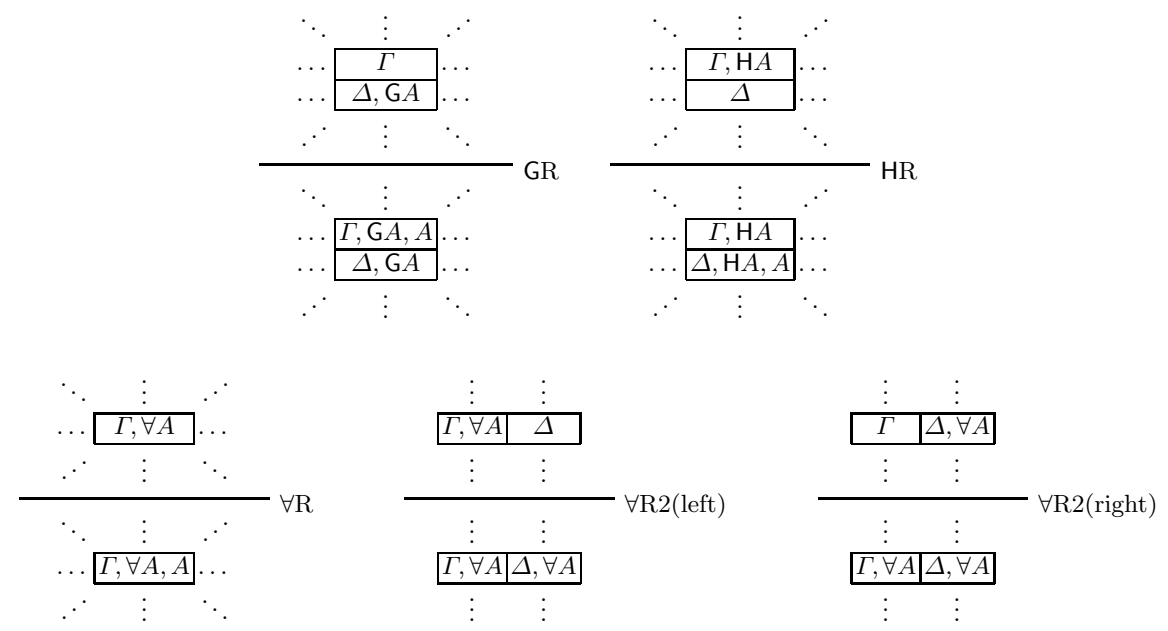

Fig. 5. General coherence-based rules.
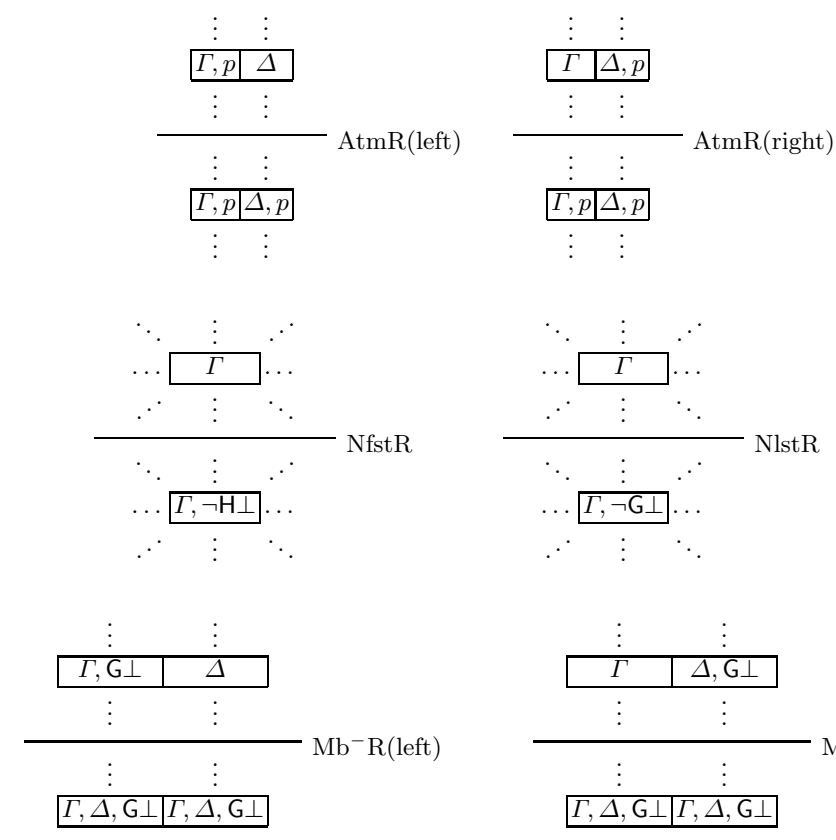

Fig. 6. Special coherence-based rules. 

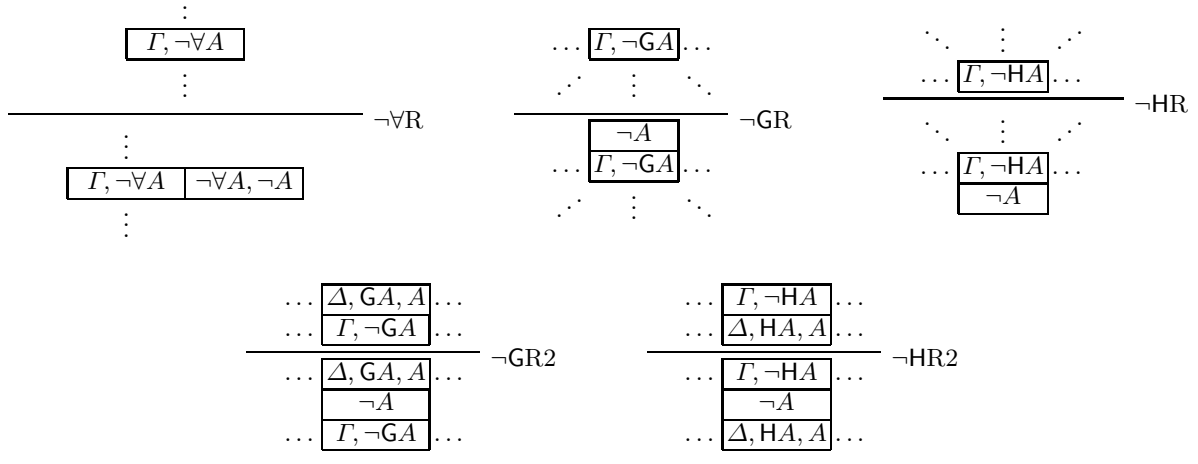

Fig. 7. General saturation-based rules.
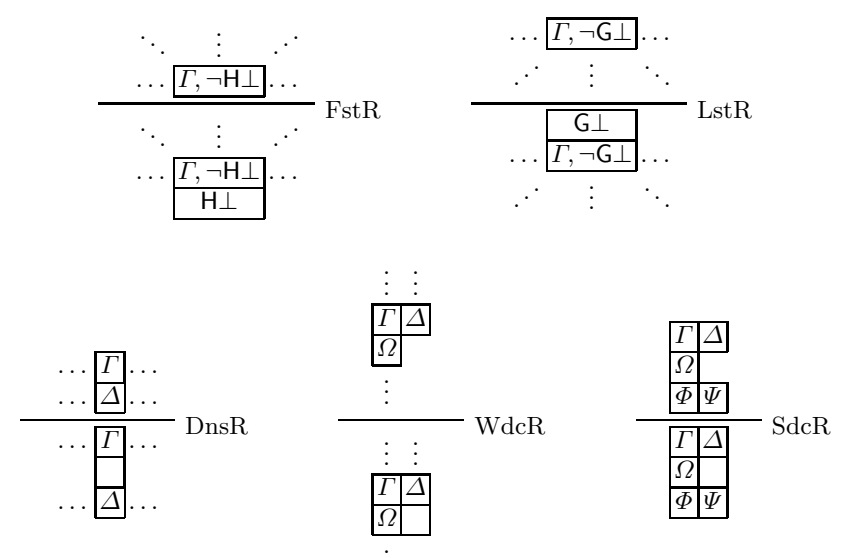

Fig. 8. Special saturation-based rules. 
- the propositional and simplification rules

DelR, CutR, ClsR, $\wedge \mathrm{R}, \neg \wedge \mathrm{R}$,

the linear-time rules ${ }^{9}$

$\mathrm{GR}, \mathrm{HR}, \neg \mathrm{GR}, \neg \mathrm{HR}, \neg \mathrm{GR} 2, \neg \mathrm{HR} 2$,

the branching rules

$\forall \mathrm{R}, \forall \mathrm{R} 2$ (left), $\forall \mathrm{R} 2$ (right), $\neg \forall \mathrm{R}$;

- rules for particular linear flows

$C \mathrm{R}$, for each condition $C$ considered;

- additional rules for particular target branching classes

- $\mathcal{D}=(\mathrm{Wdc})$

WdcR;

- $\mathcal{D}=(\mathrm{Wdc}+\mathrm{Sdc})$

WdcR, SdcR;

- $\mathcal{D}=\left(\mathrm{Wdc}+\mathrm{Sdc}+\mathrm{Mb}^{-}\right)$

WdcR, SdcR, $\mathrm{Mb}^{-} \mathrm{R}$ (left), $\mathrm{Mb}^{-} \mathrm{R}$ (right).

In the case we assume atomic harmony, the system will also contain the rules AtmR(left) and AtmR(right).

As usual, in any of these tableau systems, a tableau is a (possibly infinite) tree built from a given root by application of the tableau rules. A tableau whose root is a tuple $\Theta$ will be dubbed a tableau for $\Theta$. We say that a tableau is closed if all its branches end with the particle CLOSED. Otherwise, the tableau, as well as the corresponding branch, are said to be open. Further, a tableau is said to be exhausted if it is open but no further rules can be applied to its open branches.

In Figure 9, as an example, we show a closed tableau for the negation of the axiom WdcA.

The following is a straightforward technical result which will be useful later on.

Lemma 4. If there is a closed tableau for a given tuple $\Theta$, then:

(i) there exists a tuple $\Theta^{0}$ for which the exact same tree is also a closed tableau, such that $\Theta^{0}$ is defined at exactly the same positions as $\Theta$ and, at each defined position $p, \Theta_{p}^{0} \subseteq_{\text {fin }} \Theta_{p}$;

(ii) the exact same tree is also a closed tableau for any tuple $\Theta^{+}$defined at all the positions where $\Theta$ is defined and such that, at each defined position $p$, $\Theta_{p} \subseteq \Theta_{p}^{+}$.

Proof. For $(i)$, observe that in each rule of the system the number of "relevant" formulas (i.e., those necessary in order to make the rule applicable) in the premises is finite. The thesis follows by noticing that the number of rules applied in a closed tableau is finite. As for (ii), just observe that all the rules applied in the closed tableau for $\Theta$ can still be applied if we have as a root a tuple $\Theta^{+}$ such that all its positions extend those of $\Theta$.

\footnotetext{
${ }^{9}$ Collecting just the propositional and simplification rules, plus the linear-time rules, we get a tableau system that is essentially the same as the one described in [16].
} 


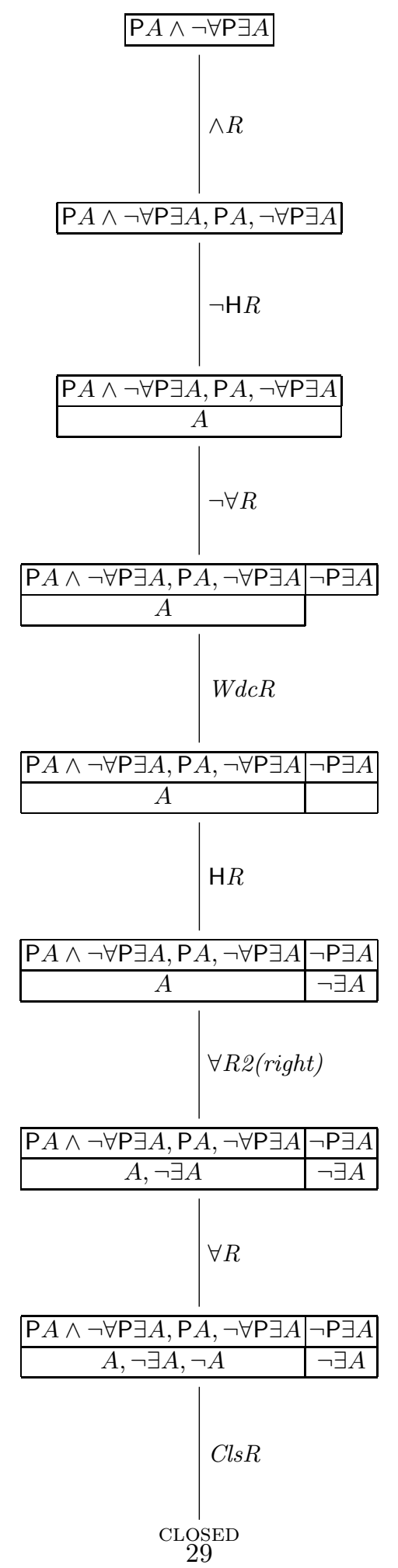

Fig. 9. A closed tableau for the negation of WdcA. 
An $\mathcal{R}(\mathcal{C}, \mathcal{D})$-tableau is a tableau built by using only rules in $\mathcal{R}(\mathcal{C}, \mathcal{D})$. Given a set $\Gamma \subseteq \mathcal{F}$, we say that it is $\mathcal{R}(\mathcal{C}, \mathcal{D})$-consistent if there is no closed $\mathcal{R}(\mathcal{C}, \mathcal{D})$ tableau for $\Gamma$. The set $\Gamma$ is further said to be maximally $\mathcal{R}(\mathcal{C}, \mathcal{D})$-consistent if either $A \in \Gamma$ or $\neg A \in \Gamma$ for every $A \in \mathcal{F}$. If $\Gamma$ is not $\mathcal{R}(\mathcal{C}, \mathcal{D})$-consistent, it will be called $\mathcal{R}(\mathcal{C}, \mathcal{D})$-inconsistent.

Theorem 7. For each class $\mathcal{C}$ of linear orders such that Udsc and Ddsc are not in $\mathcal{C}$ and each target branching class $\mathcal{D}$, the tableaux system $\mathcal{R}(\mathcal{C}, \mathcal{D})$ for the logic $\mathcal{L}(\mathcal{C}, \mathcal{D})$ is sound.

In order to show soundness (that is, if a set $\Gamma$ of formulas is inconsistent then it is not satisfiable by a $\mathcal{C}$ - $\mathcal{D}$-structure) it suffices to check, for each tableau rule, that if its numerator is satisfiable then so must be at least one of the denominators. Also this proof is routine and we thus omit it.

Theorem 8. For each class $\mathcal{C}$ of linear orders such that Udsc and Ddsc are not in $\mathcal{C}$ and each target branching class $\mathcal{D}$, the tableaux system $\mathcal{R}(\mathcal{C}, \mathcal{D})$ for the logic $\mathcal{L}(\mathcal{C}, \mathcal{D})$ is complete.

Proof. For completeness, we must prove that if a set $\Gamma$ of formulas is $\mathcal{R}(\mathcal{C}, \mathcal{D})$ consistent then it is $\mathcal{C}$ - $\mathcal{D}$-satisfiable. Taking advantage of mosaics, we will show that there is a $\mathcal{C}$ - $\mathcal{D}$-structure of mosaics for $\Gamma$. Concretely, we will define a unique $\mathcal{C}$ - $\mathcal{D}$-structure of mosaics that contains points corresponding to all $\mathcal{R}(\mathcal{C}, \mathcal{D})$ consistent sets.

Let $S=\left(S_{V}, S_{H}\right)$ be such that:

$-S_{V}$ contains precisely

- $(\Delta)$ for each maximally $\mathcal{R}(\mathcal{C}, \mathcal{D})$-consistent set $\Delta$, and

- $(\Omega, \Delta)$ for each pair of maximally $\mathcal{R}(\mathcal{C}, \mathcal{D})$-consistent sets $\Omega, \Delta$ such that there is no closed tableau for $\frac{\Delta}{\Omega}$.

$-S_{H}$ contains

- $(\Delta)$ for each maximally $\mathcal{R}(\mathcal{C}, \mathcal{D})$-consistent set $\Delta$, and

- $(\Omega, \Delta)$ for each pair of maximally $\mathcal{R}(\mathcal{C}, \mathcal{D})$-consistent sets $\Omega, \Delta$ such that there is no closed tableau for $\Omega \mid \Delta$.

As $\Gamma$ is $\mathcal{R}(\mathcal{C}, \mathcal{D})$-consistent it can be extended to a maximally $\mathcal{R}(\mathcal{C}, \mathcal{D})$-consistent set $\Gamma^{\prime}$, e.g., by considering one of the open branches of a CutR exhausted $\mathcal{C}-\mathcal{D}$ tableau for $\Gamma$. Hence, $\Gamma^{\prime}$ is a point of $S$. Therefore, all we need to show is that $S$ is indeed a $\mathcal{C}$ - $\mathcal{D}$-structure of mosaics.

The proof will be modular with respect to local, vertical, horizontal and compositional properties. Namely, one can notice that in what follows each condition $C$ will be proved to be satisfied by $S$ by using only the rules present in the systems $\mathcal{R}(\mathcal{C}, \mathcal{D})$ for those classes $\mathcal{C}$ and $\mathcal{D}$ such that a $\mathcal{C}$ - $\mathcal{D}$-structure of mosaics is required to satisfy $C$.

The first part of the proof, concerning local and vertical conditions, follows from the one in [16]; we will omit most of the details. 
Local conditions. First of all, it is easy to notice that any maximally $\mathcal{R}(\mathcal{C}, \mathcal{D})$ consistent set is a point (on $\mathcal{F}$ ). Condition L1 follows immediately from maximal consistency. For condition L2, assume $B \wedge C \in \Gamma$ and either $B \notin \Gamma$ or $C \notin \Gamma$; by using $\wedge \mathrm{R}$, one gets a closing situation, which contradicts the consistency of $\Gamma$. The other direction of L2 is proved similarly by using $\neg \wedge \mathrm{R}$ and ClsR; condition L3 follows from $\forall \mathrm{R}$ and ClsR.

Vertical coherence conditions. As in [16], by using GR, HR and ClsR, we have that each $(\Omega, \Delta) \in S_{V}$ is a vertical mosaic.

Horizontal coherence conditions. To prove that $(\Omega, \Delta) \in S_{H}$ is a horizontal mosaic, we must show that $\Omega$ and $\Delta$ are state-equivalent (property H1). We proceed by induction. As a base case, we have that if $\forall A \in \Omega$ and $\forall A \notin \Delta$ (or vice-versa, without loss of generality) then $\neg \forall A \in \Delta$. But by using $\forall \mathrm{R} 2$ (left/right) we would get to a closing situation on $\forall A$, which contradicts $(\Omega, \Delta) \in S_{H}$.

In case we assume atomic harmony, we have a further base case concerning atomic propositions. If $p \in \Omega$ and $p \notin \Delta$ (or vice-versa, without loss of generality) then $\neg p \in \Delta$. But using AtmR2(left/right) we would get to a closing situation, which again contradicts $(\Omega, \Delta) \in S_{H}$.

Then we have two step cases, for the boolean connectives $\wedge$ and $\neg$. Assume that $A$ and $B$ are state-formulas. (i) If $A \wedge B \in \Omega$ and $A \wedge B \notin \Delta$ (or vice-versa, without loss of generality) then $\neg(A \wedge B) \in \Delta$. Using $\wedge$ R we conclude that $A, B \in \Omega$ and by the induction hypothesis also $A, B \in \Delta$. But using $\neg \wedge \mathrm{R}$ both branches would get to a closing situation, which contradicts $(\Omega, \Delta) \in S_{H}$. (ii) If $\neg A \in \Omega$ and $\neg A \notin \Delta$ (or vice-versa, without loss of generality) then $A \in \Delta$. Using the induction hypothesis also $A \in \Omega$, leading to a closing situation, which contradicts $(\Omega, \Delta) \in S_{H}$.

Vertical saturation conditions. Vertical saturation conditions SV1-SV4 can be proved as in the linear case [16], by using $\neg \mathrm{GR}, \neg \mathrm{HR}, \neg \mathrm{GR} 2$ and $\neg \mathrm{HR} 2$, respectively, plus cutR to get maximally consistent sets.

Conditions on particular linear flows. In the special cases when $\mathcal{C}$ includes the properties Nlst, Lst, Nfst or Fst, we can prove that the maximally $\mathcal{R}(\mathcal{C}, \mathcal{D})$ consistent sets are FU, FB, PU or PB-points, respectively, by using NlstR, LstR, NfstR and FstR, respectively. We prove the claim for the property Nlst. Let $\Gamma$ be a maximally $\mathcal{R}(\mathcal{C}, \mathcal{D})$-consistent set, for $\mathcal{C}$ containing Nlst, and assume for the sake of contradiction that it is not an FU-point, i.e., that $\neg \mathrm{G} \perp \notin \Gamma$, which implies $\mathrm{G} \perp \in \Gamma$. By applying the rule NlstR, we get a position containing both $\mathrm{G} \perp$ and $\neg \mathrm{G} \perp$.

As further vertical saturation conditions, let us consider density (SV-Dns). Let $(\Delta, \Gamma) \in S_{V}$. Then, using DnsR, it is clear that there is also no closed tableau for $\frac{T}{\Delta}$. Hence we can use cutR to maximize and obtain a maximally consistent set $\Omega$ such that $\frac{\Gamma}{\Omega}$ can also not be closed, which guarantees, using DelR, that $(\Delta, \Omega),(\Omega, \Gamma) \in S_{V}$. 
Horizontal saturation conditions. We have to prove that $S$ satisfies SH1. Let $\Delta$ be maximally consistent and $\neg \forall \neg A \in \Delta$. Using $\neg \forall \mathrm{R}$ and cutR to maximize we get a maximally $\mathcal{R}(\mathcal{C}, \mathcal{D})$-consistent $\Omega$ such that $A \in \Omega$ and $(\Delta, \Omega) \in S_{H}$.

SWdc. Let $(\Gamma, \Delta) \in S_{H}$ and $(\Omega, \Gamma) \in S_{V}$. We first show that there is no closed tableau for $\frac{\Gamma \Delta}{\Omega}$. If this root could be closed, using Lemma 4 there would be finite subsets $\Delta_{0} \subseteq \Delta$ and $\Omega_{0} \subseteq \Omega$ such that also $\frac{\Gamma}{\Omega_{0}} \Delta_{0}$ could be closed. But $(\Gamma, \Delta) \in S_{H}$ and $(\Omega, \Gamma) \in S_{V}$ which implies that $\mathrm{P}\left(\bigwedge \Omega_{0}\right), \exists\left(\bigwedge \Delta_{0}\right) \in \Gamma$. Therefore we could also build a closed tableau for $\Gamma$ by just using $\neg \mathrm{HR}, \neg \forall \mathrm{R}$ and $\wedge \mathrm{R}$, which contradicts the consistency of $\Gamma$.

Hence, there is no closed tableau for $\frac{\Gamma \Delta}{\Omega}$. Therefore, we can use WdcR and then cutR to maximize and obtain a maximally $\mathcal{R}(\mathcal{C}, \mathcal{D})$-consistent set $\Phi$ such that $\frac{\Gamma \Delta}{\Omega \mid \Phi}$ can also not be closed, which guarantees, using DelR, that $(\Omega, \Phi) \in S_{H}$ and $(\Delta, \Phi) \in S_{V}$.

STrn. Let $(\Omega, \Gamma),(\Gamma, \Delta) \in S_{V}$. To prove that $(\Omega, \Delta) \in S_{V}$ we just need to check that there is no closed tableau for $\frac{\Delta}{\Omega}$. If this root could be closed, using Lemma 4 there would be finite subsets $\Delta_{0} \subseteq \Delta$ and $\Omega_{0} \subseteq \Omega$ such that also $\frac{\Delta_{0}}{\Omega_{0}}$ could be closed. But $(\Omega, \Gamma),(\Gamma, \Delta) \in S_{V}$ which implies that $\mathrm{P}\left(\bigwedge \Omega_{0}\right), \mathrm{F}\left(\bigwedge \Delta_{0}\right) \in \Gamma$. Therefore we could also build a closed tableau for $\Gamma$ by just using $\neg \mathrm{HR}, \neg \mathrm{GR}, \wedge \mathrm{R}$ and DelR, which contradicts the consistency of $\Gamma$.

SCon. Let $(\Delta, \Gamma),(\Omega, \Gamma) \in S_{V}$ with $\Delta \neq \Omega$. To prove that $(\Omega, \Delta) \in S_{V}$ or $(\Delta, \Omega) \in S_{V}$ we just need to check that there cannot be closed tableaux for both $\frac{\Delta}{\Omega}$ and $\frac{\Omega}{\Delta}$. If that were the case, using Lemma 4 there would be finite subsets $\Delta_{0}^{\prime}, \Delta_{0}^{\prime \prime} \subseteq \Delta$ and $\Omega_{0}^{\prime}, \Omega_{0}^{\prime \prime} \subseteq \Omega$ such that also $\frac{\Delta_{0}^{\prime}}{\Omega_{0}^{\prime}}$ and $\frac{\Omega_{0}^{\prime \prime}}{\Delta_{0}^{\prime \prime}}$ could be closed. Notice that $\Delta \neq \Omega$ and let $B$ be some formula such that $B \in \Delta$ and $B \notin \Omega$, i.e., $\neg B \in \Omega$. Let $\Delta_{0}=\Delta_{0}^{\prime} \cup \Delta_{0}^{\prime \prime}$ and $\Omega_{0}=\Omega_{0}^{\prime} \cup \Omega_{0}^{\prime \prime}$.

Since $(\Delta, \Gamma),(\Omega, \Gamma) \in S_{V}$, we have that $\mathrm{P}\left(B \wedge\left(\bigwedge \Delta_{0}\right)\right), \mathrm{P}\left(\neg B \wedge\left(\wedge \Omega_{0}\right)\right) \in \Gamma$. Therefore we could also build a closed tableau for $\Gamma$ by using $\neg \mathrm{HR}, \wedge \mathrm{R}$, $\neg \mathrm{HR} 2$, DelR, ClsR and CutR on $\mathrm{P}\left(\neg B \wedge\left(\bigwedge \Omega_{0}\right)\right)$ and $\neg B \wedge\left(\bigwedge \Omega_{0}\right)$ (see Figure 10

SSdc. The construction is similar to that for SWdc. Let $(\Gamma, \Delta),(\Phi, \Psi) \in S_{H}$ and $(\Omega, \Gamma),(\Phi, \Omega) \in S_{V}$. We first show that there is no closed tableau for $\Gamma \Delta$

$\Omega \quad$. For the sake of contradiction, let us assume that this root can be closed. Then, by using Lemma 4 there would be finite subsets $\Gamma_{0} \subseteq \Gamma$, $\Delta_{0} \subseteq \Delta, \Phi_{0} \subseteq \Phi$ and $\Psi_{0} \subseteq \Psi$ such that also $\begin{array}{ll}\frac{T_{0} \mid \Delta_{0}}{\Omega} \\ \frac{\Omega}{\Phi_{0} \mid} \Psi_{0}\end{array}$ could be closed. But $(\Gamma, \Delta),(\Phi, \Psi) \in S_{H}$ and $(\Omega, \Gamma),(\Phi, \Omega) \in S_{V}$ imply that $\mathrm{F}\left(\bigwedge \Gamma_{0} \wedge\right.$ $\left.\exists \wedge \Delta_{0}\right), \mathrm{P}\left(\bigwedge \Phi_{0} \wedge \exists \wedge \Psi_{0}\right) \in \Omega$. Therefore we could also build a closed tableau 
for $\Omega$ by just using $\neg \mathrm{HR}, \neg \mathrm{GR}, \neg \forall \mathrm{R}$ and $\wedge \mathrm{R}$, which contradicts the consistency of $\Omega$.

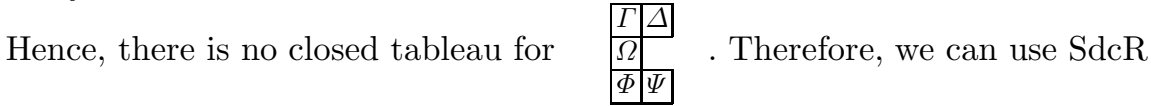
and then cutR to maximize and obtain a maximally $\mathcal{R}(\mathcal{C}, \mathcal{D})$-consistent set

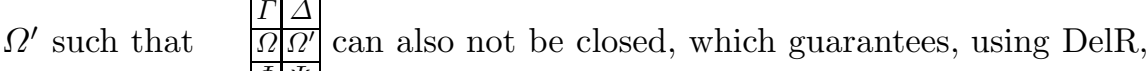
$\Phi$ 业

that $\left(\Omega, \Omega^{\prime}\right) \in S_{H}$ and $\left(\Psi, \Omega^{\prime}\right),\left(\Omega^{\prime}, \Delta\right) \in S_{V}$.

$\mathrm{SMb}^{-}$. Let $\Gamma, \Delta$ be distinct maximally $\mathcal{R}(\mathcal{C}, \mathcal{D})$-consistent sets such that $\mathrm{G} \perp \in \Gamma$ (without loss of generality). We must show that $(\Gamma, \Delta) \notin S_{H}$. It suffices to produce a closed tableau for $\Gamma \Delta$. Notice that as $\Gamma \neq \Delta$, there exists $B$ such that $B \in \Gamma$ and $B \notin \Delta$, i.e., $\neg B \in \Delta$. Thus, using $\mathrm{Mb}^{-} \mathrm{R}$ (left/right) we get into a closing situation.

Clearly, the unrestricted CutR rule is a problem with respect to implementations, also preventing us from obtaining tableau-based decision procedures for the logics. However, in certain cases, namely when we consider the target branching class $\mathcal{D}=()$, it is possible to use only analytical instances of the cut rule. Let $\Gamma$ be a finite set of formulas. Given a tableau system $\mathcal{R}(\mathcal{C}, \mathcal{D})$, we can define its analytic restriction with respect to $\Gamma$ as the system $\mathcal{R}_{\Gamma}(\mathcal{C}, \mathcal{D})$ obtained from $\mathcal{R}(\mathcal{C}, \mathcal{D})$ by replacing CutR with a version of the rule that can only introduce a formula $A \in \Lambda$, where $\Lambda$ is the smallest $(\mathcal{C}, \mathcal{D})$-closed set of formulas containing $\Gamma$. Note that in this analytic restriction, discreteness could be also considered, e.g., by including in the system the following two rules:
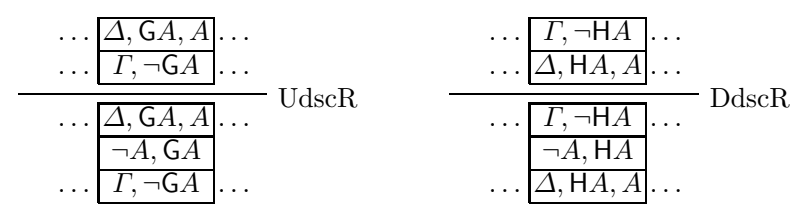

Theorem 9. Let $C$ be a class of linear orders and $\Gamma$ a finite set of formulas. If $\Gamma$ is $\mathcal{R}_{\Gamma}(\mathcal{C},())$-consistent then it is $\mathcal{C}$-()-satisfiable.

Proof. By following the proof of Theorem 8, we can modify the definition of $S$ by requiring the points of $S$ to be $\mathcal{R}_{\Gamma}(\mathcal{C},())$-consistent sets maximal in $\Lambda$, where $\Lambda$ is the smallest $(\mathcal{C},())$-closed set of formulas containing $\Gamma$. The assert follows from noticing that in the proof of Theorem 8 , for showing only the conditions satisfied by $\mathcal{C}$-()-structures of mosaics, non-analytic cuts are never used and that all the relevant rules are such that if all the formulas in the numerator are in $\Lambda$ then the same happens to all the formulas in the denominator. Finally, we notice that the restricted version of the cut is enough for maximalizing sets, when required by the proof, with respect to $\Lambda$.

Theorem 9 gives the completeness of the analytic system with respect to the $\operatorname{logic} \mathcal{L}(\mathcal{C},())$. Its soundness is a trivial consequence of Theorem 7 . 


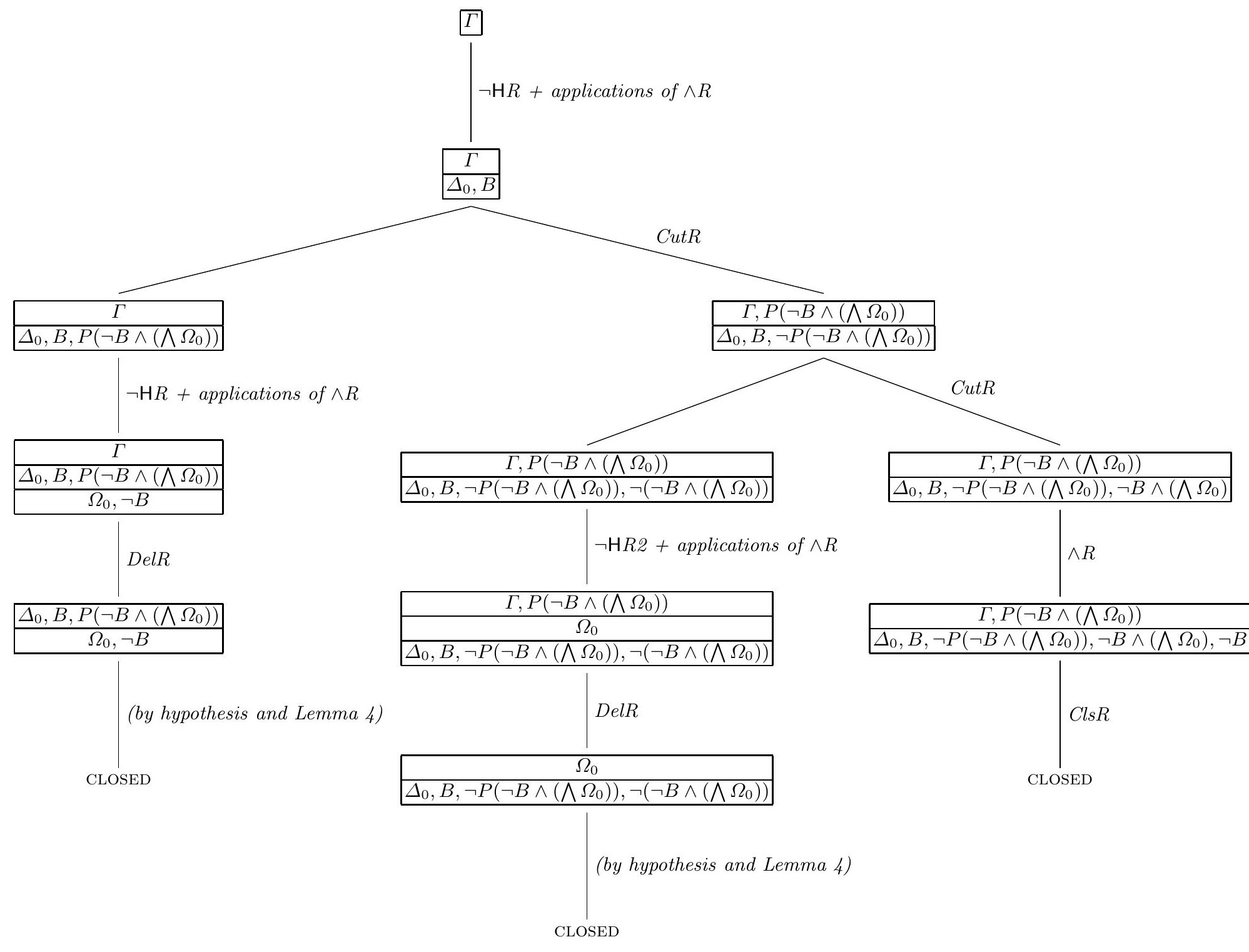

Fig. 10. A tableau for the proof of the property SCon. 
Notice, instead, that, for proving conditions SWdc, STrans, SConn and SSdc in Theorem 8 (i.e., when we consider target branching classes different from the basic one), we make essential use of unboundedly complex formulas and that in proving SConn we even use cuts on such formulas. This prevents us from obtaining any obvious corresponding analyticity result.

\subsection{Decidability via mosaics}

Another interesting application of the mosaic technique, which we pursue here, is in proving the decidability of a given logic and in obtaining an asymptotic upper-bound on its decision problem. We will show how mosaics can be used in order to prove decidability of the logics considered in the paper in the particular case of basic structures. The proof follows the idea of the decidability proofs in [16] and [21].

Theorem 10. The problem of checking satisfiability of formulas in the logic of $\mathcal{C}$-()-frames, for $\mathcal{C}$ a class of linear orders, is decidable.

Proof. Let $A$ be a satisfiable formula. By following the same construction as in the proof of right-to-left direction of Theorem 2 (just consider the finite set $\Gamma$ as consisting only of $A$ ), we can define a structure of mosaics for $A$ on a finite labeling set $\Lambda$.

Since $\Lambda$ is finite, the number of possible mosaics, and thus of structures of mosaics, on it is also finite. Given that checking coherence and saturation conditions is decidable, we can take each pair of sets of mosaics in turn and check whether it is a $\mathcal{C}$-()-structure of mosaics for $A$.

We can obtain an asymptotic upper bound by observing that the cardinality of the set $\Lambda$, as defined in the proof of Theorem 10, is $O(n)$, where $n$ is the complexity of $A$. It follows that the number of possible mosaics on that set is $O\left(2^{n}\right)$ and the number of structures of mosaics is $O\left(2^{2^{n}}\right)$. Coherence and saturation conditions can be checked in polynomial time.

It is easy to see that the argument in the proof of Theorem 10 does not extend to the logics $\mathcal{L}(\mathcal{C}, \mathcal{D})$ for $\mathcal{D} \neq()$. Namely, when there is some interaction between the vertical and the horizontal components, the simple translation of a model into sets of mosaics described in the proof above produces a structure that does not necessarily satisfy all the saturation conditions required. This is of course also related to the results of Section 4.2, where an analytic version of the tableau system has been proven to be sound and complete only in the case of the target branching class being the basic one. The possibility of cutting with respect to the full language $\mathcal{F}$ is necessary there in order to get tableaux completeness for logics $\mathcal{L}(\mathcal{C}, \mathcal{D})$ where $\mathcal{D} \neq()$; analogously, considering mosaics defined on $\Lambda=\mathcal{F}$ would allow the construction of the proof of Theorem 10 to provide a structure of mosaics satisfying all the saturation conditions (but then the result of decidability would not follow since the number of possible mosaics on $\mathcal{F}$ is infinite). 
In order to get a proof of decidability based on mosaics also for the other classes of logics ${ }^{10}$, it might be useful to consider a more complex, and branchingoriented, notion of mosaic (more on this in Section 5).

The proof of decidability given here seems extremely appealing because of its simplicity. We observe that it should also be possible to define a decision procedure for the $\mathcal{C}$-()-logics based on the tableau system of Section 4.2 , by exploiting analyticity of the cut rule in that case and properly avoiding the repeated curing of the same defect.

\section{Conclusions}

We have proposed an extension of the mosaic method from a class of linear temporal logics to a two-dimensional logic obtained by adding an "orthogonal" S5-like component, and we have treated several applications of the method. Namely we have shown how the mosaic techniques can be used to prove completeness for the corresponding Hilbert-style axiomatization, to define a sound and complete tableau system and to obtain a decision procedure for the logic considered as well as to establish an asymptotic upper-bound on its complexity.

In [16] the mosaic method has been proposed for the general linear-time logics together with some variants capturing particular (i.e., dense, discrete, bounded/unbounded) linear flows of time. The approach presented here can be seen as a conservative extension of that method, in the sense that our presentation is modularized with respect to a vertical (linear-time) and a horizontal (S5-like) component, in such a way that the first one consists of definitions and proofs just imported from [16].

We have also considered the possibility of having interactions between the two components, in order to treat logics that capture the idea of branchingtime. To that aim, our treatment has been parameterized along both the two components: with respect to the class of linear orders considered, ranging from the general to more specific ones (i.e., dense, discrete, etc.), and with respect to the class of branching structures, according to a hierarchy leading from the basic ones, where the two orthogonal components are independent, to "more branching" ones, like the Ockhamist structures of, e.g., [31].

Namely, by letting $\mathcal{C}$ range over classes of linear orders and $\mathcal{D}$ over sets of branching properties, we have considered a broad class of Ockhamist branchingtime logics $\mathcal{L}(\mathcal{C}, \mathcal{D})$ and defined for them, by means of a fully modular presentation, an extension of the mosaic method. Indeed, this two-dimensional view allows for dealing, in a clear way, with the logics defined over Ockhamist structures where all the vertical components are in the same class of linear orders, or, which is equivalent, to the class of bundled trees such that all the paths are in the same class of linear orders. However while, as long as the vertical and

\footnotetext{
${ }^{10}$ We recall that decidability of the logic $\mathcal{L}\left((),\left(W d c+S d c+M b^{-}\right)\right)$is proved in [1], using Rabin's Theorem [20], which states the decidability of the monadic second order theory of infinite binary trees. In [7], such a proof is adapted to the case with atomic harmony.
} 
the horizontal component behave independently, all the results in [16] (proof of Hilbert-style completeness, definition of a complete tableau system and proof of decidability) can be proved to propagate, in the case when interactions between the two components are considered we have some restrictions: a tableau system can only be defined by allowing a non-analytic version of the cut rule and thus the proof of decidability does not apply.

We believe that our approach, presented here with a focus on temporal logics, can be seen as more generally suitable for dealing with many-dimensional modal logics without interactions between the dimensions [9]. In such a context, mosaics can be seen as an alternative to other techniques typically used in order to get (the transfer of) decidability or completeness results, such as fibring [8].

Further work is required in order to capture properly, i.e., in a way that allows for proving decidability, also logics where interactions are considered, such as the branching-time logics seen in this paper or several logics of knowledge and time $[5,13]$ which present similar interaction frame properties. Our future research will consider the possibility of having a more complex notion of mosaic, in some way taking into account, already in the definition of the basic components of our structures, the possible interactions between the dimensions (i.e., in the case of temporal logics, their branching nature). As an example, we recall the treatment in [21], where the decidability of a logic defined over rectangular frames consisting of the cross product of a (vertical) linear order and a (horizontal) set of worlds, is proved by using mosaics that are pairs of horizontal segments of points. We are aware that, as a trade-off, an approach of this kind would probably compromise (at least part of) the desirable modularity properties, with respect to the linear treatment of [16], that the presentation proposed here enjoys.

Acknowledgments. The authors are grateful to Andrea Masini and Alberto Zanardo for several fruitful discussions on the subject of this paper, and to the anonymous referee for valuable remarks on an earlier version of this paper.

\section{References}

1. J. P. Burgess. Logic and time. J. Symb. Log., 44(4):566-582, 1979.

2. J. P. Burgess. Decidability for branching time. Studia Logica, 39:203-218, 1980.

3. J. P. Burgess. Axioms for tense logic. I. "Since" and "until". Notre Dame J. Formal Logic, 23(4):367-374, 1982.

4. M. C. Di Maio, and A. Zanardo. Synchronized Histories in Prior-Thomason Representation of Branching Time. In D. M. Gabbay and H. J. Ohlbach, editors, ICTL '94, LNCS 827, pages 265-282. Springer, 1994.

5. R. Fagin, J. Halpern, Y. Moses, and M. Vardi. Reasoning about knowledge. MIT Press, 1995

6. D. M. Gabbay. An irreflexivity lemma with applications to axiomatizations of conditions on tense frames. Aspects of philosophical logic, 67-89., 1981.

7. D. M. Gabbay, I. Hodkinson, and M. Reynolds. Temporal logic (vol. 1): mathematical foundations and computational aspects. Oxford University Press, 1994.

8. D. M. Gabbay, V. B. Shehtman. Products of Modal Logics, Part 1. Logic Journal of the IGPL, 6(1):73-146, 1998. 
9. D. M. Gabbay, A. Kurucz, F. Wolter, and M. Zakharyaschev. Many-dimensional modal logics: theory and applications. Studies in Logic, 148. Elsevier Science, 2003.

10. R. I. Goldblatt. Logics of Time and Computation. CSLI Lecture Notes, 1987.

11. R. Goré. Tableau methods for modal and temporal logics. In Handbook of Tableau Methods, pages 297-396. Kluwer, 1999.

12. V. Goranko, A.Zanardo. From Linear to Branching-Time Temporal Logics: Transfer of Semantics and Definability Logic Journal of the IGPL, 15(1):53-76, 2007.

13. J. Y. Halpern, R. Van der Meyden, and M. Y. Vardi. Complete Axiomatizations for Reasoning about Knowledge and Time. SIAM J. Comput., 33(3):674-703, 2004.

14. R. Hirsch, I. Hodkinson, M. Marx, S. Mikulás, and M. Reynolds. Mosaics and step-by-step. Remarks on "A modal logic of relations". In Logic at Work. Essays Dedicated to the Memory of Helena Rasiowa, pages 158-167. Springer, 1999.

15. A. Kurucz. Combining modal logics. In J. van Benthem, P. Blackburn, F. Wolter, editors, Handbook of Modal Logic, pages 869-924. Elsevier, 2007.

16. M. Marx, S. Mikulás, and M. Reynolds. The mosaic method for temporal logics. In R. Dyckhoff, editor, TABLEAUX, LNCS 1847, pages 324-340. Springer, 2000.

17. S. Mikulás. Taming first-order logic. Journal of the IGPL, 6(2):305-316, 1998.

18. I. Németi. Free Algebras and Decidability in Algebraic Logic. PhD thesis, Hungarian Academy of Sciences, Budapest, 1986.

19. I. Németi. Decidable versions of first order logic and cylindric-relativized set algebras. In Logic Colloquium '92, pages 171-241. CSLI Publications, 1995.

20. M. O. Rabin. Decidability of second-order theories and automata on infinite trees. Trans. Amer. Math. Soc., 141:1-35, 1969.

21. M. Reynolds. A decidable temporal logic of parallelism. Notre Dame Journal of Formal Logic, 38:419-436, 1996.

22. M. Reynolds. Axioms for branching time. J. Log. Comput., 12(4):679-697, 2002.

23. M. Reynolds. The complexity of the temporal logic with "until" over general linear time. J. Comput. Syst. Sci., 66(2):393-426, 2003.

24. M. Reynolds. Dense time reasoning via mosaics. In TIME '09, pages $3-10$. IEEE Computer Society, 2009.

25. M. Reynolds. The complexity of decision problems for linear temporal logics. Journal of Studies in Logic, 3(1):19-50, 2010.

26. M. Reynolds. The complexity of temporal logic over the reals. Annals of Pure and Applied Logic, 161:1063-1096, 2010.

27. R. H. Thomason. Combinations of tense and modality. In Handbook of Philosophical Logic: Extensions of Classical Logic, pages 135-165. Reidel, 1984.

28. D. Van Dalen. Logic and structure. Springer, 1994.

29. Y. Venema and M. Marx. A modal logic of relations. In Logic at Work: Essays Dedicated to the Memory of Helena Rasiowa. Springer, 1999.

30. A. Zanardo. A finite axiomatization of the set of strongly valid Ockhamist formulas. Journal of Philosophical Logic, 14:447-468, 1985.

31. A. Zanardo. Branching-time logic with quantification over branches: The point of view of modal logic. Journal of Symbolic Logic, 61(1):1-39, 1996. 